TRANSACTIONS OF THE

AMERICAN MATHEMATICAL SOCIETY

Volume 361, Number 3, March 2009, Pages 1463-1493

S 0002-9947(08)04703-X

Article electronically published on October 17, 2008

\title{
MODULAR INVARIANCE AND TWISTED CANCELLATIONS OF CHARACTERISTIC NUMBERS
}

\author{
QINGTAO CHEN AND FEI HAN
}

\begin{abstract}
By studying modular invariance properties of some characteristic forms, which are related to elliptic genera, we obtain twisted cancellation formulas for characteristic forms. We apply these twisted cancellation formulas to study divisibilities on spin manifolds and congruences on $\operatorname{spin}^{c}$ manifolds. In particular, we obtain twisted Rokhlin congruences for $8 k+4$ dimensional spin $^{c}$ manifolds.
\end{abstract}

\section{INTRODUCTION}

Let $M$ be a 12 dimensional smooth Riemannian manifold. A beautiful relation between the top degree components of the Hirzebruch $\widehat{L}$-form and $\widehat{A}$-form of $M$ (see (2.1) and (2.2) below for their definitions) was shown by Alvarez-Gaumé and Witten 1 as a gravitational anomaly cancellation formula as follows:

$$
\left\{\widehat{L}\left(T M, \nabla^{T M}\right)\right\}^{(12)}=\left\{8 \widehat{A}\left(T M, \nabla^{T M}\right) \operatorname{ch}\left(T_{\mathbf{C}} M, \nabla^{T_{\mathbf{C}} M}\right)-32 \widehat{A}\left(T M, \nabla^{T M}\right)\right\}^{(12)}
$$

where $T_{\mathbf{C}} M$ denotes the complexification of $T M$ and $\nabla^{T_{\mathbf{C}} M}$ is canonically induced from $\nabla^{T M}$, the Levi-Civita connection associated to the Riemannian structure of $M ; \operatorname{ch}\left(T_{\mathbf{C}} M, \nabla^{T_{\mathbf{C}} M}\right)$ denotes the Chern character form associated to $\left(T_{\mathbf{C}} M, \nabla^{T_{\mathbf{C}} M}\right)$ (cf. [22]). This gravitational anomaly cancellation formula, which they called the miraculous cancellation formula, was derived from very non-trivial computations.

(1.1) is generalized by Kefeng Liu [16] to arbitrary $8 k+4$ dimensional manifolds by developing modular invariance properties of characteristic forms. In [16], he proved that for each $8 k+4$ dimensional smooth Riemannian manifold $M$ the following identity holds:

$$
\left\{\widehat{L}\left(T M, \nabla^{T M}\right)\right\}^{(8 k+4)}=8 \sum_{r=0}^{k} 2^{6 k-6 r} h_{r}\left(T_{\mathbf{C}} M\right) .
$$

In (1.2), each $h_{r}\left(T_{\mathbf{C}} M\right)$ is a differential form

$$
\left\{\widehat{A}\left(T M, \nabla^{T M}\right) \operatorname{ch}\left(b_{r}\left(T_{\mathbf{C}} M\right), \nabla^{b_{r}\left(T_{\mathbf{C}} M\right)}\right)\right\}^{(8 k+4)},
$$

where $b_{r}\left(T_{\mathbf{C}} M\right) \in K O(M) \otimes \mathbf{C}, 0 \leq r \leq k$, can be derived canonically from $T M$. When the manifold is closed and spin, according to the Atiyah-Hirzebruch divisibility [3], $\left\langle h_{r}\left(T_{\mathbf{C}} M\right),[M]\right\rangle$ are all even numbers. Therefore from (1.2) and the Hirzebruch signature theorem [11, one easily obtains the Ochanine divisibility [19],

Received by the editors February 16, 2007.

2000 Mathematics Subject Classification. Primary 58J26; Secondary 58J20.

(C) 2008 American Mathematical Society
Reverts to public domain 28 years from publication 1463 
which says that the signature of an $8 k+4$ dimensional smooth closed spin manifold is divisible by 16 . This shows us how miraculous cancellation formulas imply divisibility of characteristic numbers. The author also provides a similar miraculous cancellation formula for $8 k$ dimensional manifolds. However this $8 k$ dimensional cancellation formula does not imply divisibility results. Liu's formula refines the arguments of Hirzebruch [10] and Landweber [14, who deduce the Ochanine divisibility by using the ideas of elliptic genus, to the level of differential forms. See [16] for details.

Liu's method is taken over in [8, 9, to study the Ochanine congruence and the Finashin congruence. The authors show that there exist more general cancellation formulas with an extra complex line bundle involved, which generalizes Liu's formula (1.2) and turn out to be efficient for studying congruence phenomena on $\operatorname{spin}^{c}$ and $\operatorname{pin}^{-}$manifolds. Let $\left(\xi, \nabla^{\xi}\right)$ be a real oriented Euclidean plane bundle, or equivalently a complex line bundle on $M$ with Euler form $c=e\left(\xi, \nabla^{\xi}\right)$. For each $8 k+4$ dimensional smooth Riemannian manifold $M$, they obtain that

$$
\left\{\frac{\widehat{L}\left(T M, \nabla^{T M}\right)}{\cosh ^{2}\left(\frac{c}{2}\right)}\right\}^{(8 k+4)}=8 \sum_{r=0}^{k} 2^{6 k-6 r} h_{r}\left(T_{\mathbf{C}} M, \xi_{\mathbf{C}}\right) .
$$

In (1.3), each $h_{r}\left(T_{\mathbf{C}} M, \xi_{\mathbf{C}}\right)$ is a differential form

$$
\left\{\widehat{A}\left(T M, \nabla^{T M}\right) \operatorname{ch}\left(b_{r}\left(T_{\mathbf{C}} M, \xi_{\mathbf{C}}\right), \nabla^{b_{r}\left(T_{\mathbf{C}} M, \xi_{\mathbf{C}}\right)}\right) \cosh \left(\frac{c}{2}\right)\right\}^{(8 k+4)},
$$

where $\xi_{\mathbf{C}}$ is the complexification of $\xi$ and $b_{r}\left(T_{\mathbf{C}} M, \xi_{\mathbf{C}}\right) \in K O(M) \otimes \mathbf{C}, 0 \leq r \leq k$, can be derived canonically from $T M$ and $\xi$.

As an application of the general cancellation formula (1.3), when $M$ is closed and $\operatorname{spin}^{c}$ and $B$ is an $8 k+2$ dimensional oriented submanifold of $M$ such that $[B] \in H_{8 k+2}(M, Z)$ is dual to $w_{2}(T M)$, the authors deduce in an analytic way that $(\operatorname{Sig}(M)-\operatorname{Sig}(B \bullet B))$ is divisible by 8 by using the Atiyah-Singer index theorem for spin $^{c}$ manifolds, where $(B \bullet B)$ denotes the self-intersection of $B$ in $M$. Moreover, they [9] show that

$$
\begin{aligned}
& \frac{\operatorname{Sig}(M)-\operatorname{Sig}(B \bullet B)}{8} \\
\equiv & \int_{M} \widehat{A}\left(T M, \nabla^{T M}\right) \operatorname{ch}\left(b_{k}\left(T_{\mathbf{C}} M+\mathbf{C}^{2}-\xi_{\mathbf{C}}, \mathbf{C}^{2}\right)\right) \cosh \left(\frac{c}{2}\right) \\
\equiv & \operatorname{ind}_{2}\left(b_{k}\left(T B+\mathbf{R}^{2}, \mathbf{R}^{2}\right)\right) \bmod 2,
\end{aligned}
$$

which is the analytic version of the Ochanine congruence obtained in [18. Formula (1.3) has interesting applications for studying the Ochanine congruence and the Finashin congruence (cf. [19], 6]). We refer interested readers to 9] for details. This shows us again how cancellation formulas imply divisibility and congruence results.

Looking at these cancellation formulas as well as the divisibilities and congruences induced by them, one asks if there exist more cancellation formulas like (1.2) and (1.3) and consequently exist more divisibilities and congruences for characteristic numbers. We show in this article some results in this direction.

Let $d+d^{*}: \Gamma\left(\Lambda T^{*} M\right) \rightarrow \Gamma\left(\Lambda T^{*} M\right)$ be the signature operator, the index of which is the signature $\operatorname{Sig}(M)$ of the manifold. It's known that ind $\left(d+d^{*}\right)=\int_{M} \widehat{L}(M)$. If 
$W$ is another complex vector bundle over $M$, one can study the twisted signature operator on the twisted signature complex, $\left(d+d^{*}\right) \otimes W: \Gamma\left(\Lambda T^{*} M \otimes W\right) \rightarrow$ $\Gamma\left(\Lambda T^{*} M \otimes W\right)$, which has $\operatorname{ind}\left(\left(d+d^{*}\right) \otimes W\right)=\int_{M} \widehat{L}(M) \operatorname{ch}(W)$. Let $\operatorname{Sig}(M, W):=$ $\operatorname{ind}\left(\left(d+d^{*}\right) \otimes W\right)$. The twisted signature complex is important in the original proof of the Atiyah-Singer index theorem. Here we are using the $\widehat{L}$-form but not its stable version the $L$-form (its characteristic series is $\frac{x}{\tanh x}$ ) because $\int_{M} \widehat{L}(M) \operatorname{ch}(W)$ gives the twisted signature $\operatorname{Sig}(M, W)$. In this paper, we are going to discuss the divisibilities (congruences respectively) of $\operatorname{Sig}\left(M, T_{\mathbf{C}} M\right)$ and $\operatorname{Sig}\left(M, T_{\mathbf{C}} M \otimes T_{\mathbf{C}} M\right)$ on spin ( $\operatorname{spin}^{c}$ respectively) manifolds, which means we will study the characteristic numbers $\left\langle\widehat{L}(T M) \operatorname{ch}\left(T_{\mathbf{C}} M\right),[M]\right\rangle,\left\langle\widehat{L}(T M) \operatorname{ch}\left(T_{\mathbf{C}} M \otimes T_{\mathbf{C}} M\right),[M]\right\rangle$.

To be more precise, still applying modular invariance of characteristic forms [16, we obtain a bunch of interesting twisted cancellation formulas for characteristic forms (with or without a twisting complex line bundle). When these new cancellation formulas are applied to $8 k$ and $8 k+4$ dimensional closed spin manifolds, we find some hidden divisibilities of the characteristic numbers $\left\langle\widehat{L}(T M) \operatorname{ch}\left(T_{\mathbf{C}} M\right),[M]\right\rangle,\left\langle\widehat{L}(T M) \operatorname{ch}\left(T_{\mathbf{C}} M \otimes T_{\mathbf{C}} M\right),[M]\right\rangle$ and some of their linear combinations.

The divisibilities of $\operatorname{Sig}\left(M, T_{\mathbf{C}} M\right)$ for $8 k$ and $8 k+4$ dimensional spin manifolds were already obtained by Hirzebruch [12] by studying elliptic genera. Our cancellation formulas supply an interesting approach for proving the Hirzebruch divisibilities. Moreover we are able to construct examples to show that the Hirzebruch divisibilities are best possible. On the other hand, the divisibilities of $\operatorname{Sig}\left(M, T_{\mathbf{C}} M \otimes\right.$ $\left.T_{\mathbf{C}} M\right)$ for $8 k$ and $8 k+4$ dimensional spin manifolds induced by our cancellation formulas look new in the literature. We are also able to construct examples to show that these divisibilities are best possible. Let $d_{s}:=d+d^{*}$ be the signature operator. The twisted signature operator $d_{s} \otimes T_{\mathbf{C}} M$ has already been proved to be rigid by using the Witten rigidity theorem; however nobody has been able to give a direct proof without using it (cf. 17]). Our divisibilities are still applications of modular invariance. It would also be interesting to find out a direct proof of these divisibilities for the twisted signatures.

On the other hand, when we apply our cancellation formulas with a twisted complex line bundle to $\operatorname{spin}^{c}$ manifolds, we obtain some congruence results about the twisted signature $\operatorname{Sig}\left(M, T_{\mathbf{C}} M\right)$, which in dimension $8 k+4$ give twisted Rokhlin congruence formulas.

The rest of the article is organized as follows. We list the twisted cancellation formulas in Section 2 and postpone their proofs to Section 5. In Section 3, we apply our twisted cancellation formulas to spin manifolds and obtain divisibilities for the twisted signatures. Then in Section 4, the twisted cancellation formulas are applied to $\operatorname{spin}^{c}$ manifolds and particularly induce twisted Rokhlin congruence formulas for $8 k+4$ dimensional $\operatorname{spin}^{c}$ manifolds.

\section{TWisted CANCELLATION FORMULAS}

In this section, we first present some basic geometric data and then list our twisted cancellation formulas.

Let $M$ be a smooth Riemannian manifold. Let $\nabla^{T M}$ be the associated LeviCivita connection and $R^{T M}=\nabla^{T M, 2}$ the curvature of $\nabla^{T M} . \nabla^{T M}$ extended canonically to a Hermitian connection $\nabla^{T_{\mathbf{C}} M}$ on $T_{\mathbf{C}} M=T M \otimes \mathbf{C}$. 
Let $\widehat{A}\left(T M, \nabla^{T M}\right), \widehat{L}\left(T M, \nabla^{T M}\right)$ be the Hirzebruch characteristic forms defined by

$$
\begin{aligned}
& \widehat{A}\left(T M, \nabla^{T M}\right)=\operatorname{det}^{1 / 2}\left(\frac{\frac{\sqrt{-1}}{4 \pi} R^{T M}}{\sinh \left(\frac{\sqrt{-1}}{4 \pi} R^{T M}\right)}\right), \\
& \widehat{L}\left(T M, \nabla^{T M}\right)=\operatorname{det}^{1 / 2}\left(\frac{\frac{\sqrt{-1}}{2 \pi} R^{T M}}{\tanh \left(\frac{\sqrt{-1}}{4 \pi} R^{T M}\right)}\right) .
\end{aligned}
$$

Let $E, F$ be two Hermitian vector bundles over $M$ carrying Hermitian connections $\nabla^{E}, \nabla^{F}$ respectively. Let $R^{E}=\nabla^{E, 2}$ (resp. $R^{F}=\nabla^{F, 2}$ ) be the curvature of $\nabla^{E}$ (resp. $\nabla^{F}$ ). If we set the formal difference $G=E-F$, then $G$ carries an induced Hermitian connection $\nabla^{G}$ in an obvious sense. We define the associated Chern character form as

$$
\operatorname{ch}\left(G, \nabla^{G}\right)=\operatorname{tr}\left[\exp \left(\frac{\sqrt{-1}}{2 \pi} R^{E}\right)\right]-\operatorname{tr}\left[\exp \left(\frac{\sqrt{-1}}{2 \pi} R^{F}\right)\right] .
$$

In the rest of the paper, where there will be no confusion about the Hermitian connection $\nabla^{E}$ on the Hermitian vector bundle $E$, we will write $\operatorname{simply} \operatorname{ch}(E)$ for the associated Chern character form.

Let $\xi$ be a rank two real oriented Euclidean vector bundle, or equivalently a complex line bundle, over $M$ carrying a Euclidean connection $\nabla^{\xi}$.

If $E$ is a complex vector bundle over $M$, set $\widetilde{E}=E-\mathbf{C}^{\mathrm{rk}(E)}$.

Let $q=e^{2 \pi \sqrt{-1} \tau}$ with $\tau \in \mathbf{H}$ be the upper half complex plane.

We introduce four elements (cf. [16, [9]) in $K(M)\left[\left[q^{\frac{1}{2}}\right]\right]$ which consist of formal power series in $q^{\frac{1}{2}}$ with coefficients in the $K$-group of $M$,

$$
\begin{aligned}
& \Theta_{1}\left(T_{\mathbf{C}} M\right)=\bigotimes_{n=1}^{\infty} S_{q^{n}}\left(\widetilde{T_{\mathbf{C}} M}\right) \otimes \bigotimes_{m=1}^{\infty} \Lambda_{q^{m}}\left(\widetilde{T_{\mathbf{C}} M}\right) \\
& \Theta_{2}\left(T_{\mathbf{C}} M\right)=\bigotimes_{n=1}^{\infty} S_{q^{n}}\left(\widetilde{T_{\mathbf{C}} M}\right) \otimes \bigotimes_{m=1}^{\infty} \Lambda_{-q^{m-\frac{1}{2}}}\left(\widetilde{T_{\mathbf{C}} M}\right) \\
& \Theta_{1}\left(T_{\mathbf{C}} M, \xi_{\mathbf{C}}\right)=\bigotimes_{n=1}^{\infty} S_{q^{n}}\left(\widetilde{T_{\mathbf{C}} M}\right) \otimes \bigotimes_{m=1}^{\infty} \Lambda_{q^{m}}\left(\widetilde{T_{\mathbf{C}} M}-2 \widetilde{\xi_{\mathbf{C}}}\right) \\
& \otimes \bigotimes_{r=1}^{\infty} \Lambda_{q^{r-\frac{1}{2}}}\left(\widetilde{\xi_{\mathbf{C}}}\right) \otimes \bigotimes_{s=1}^{\infty} \Lambda_{-q^{s-\frac{1}{2}}}\left(\widetilde{\xi_{\mathbf{C}}}\right) \\
& \Theta_{2}\left(T_{\mathbf{C}} M, \xi_{\mathbf{C}}\right)=\bigotimes_{n=1}^{\infty} S_{q^{n}}\left(\widetilde{T_{\mathbf{C}} M}\right) \otimes \bigotimes_{m=1}^{\infty} \Lambda_{-q^{m-\frac{1}{2}}}\left(\widetilde{T_{\mathbf{C}} M}-2 \widetilde{\xi_{\mathbf{C}}}\right) \\
& \otimes \bigotimes_{r=1}^{\infty} \Lambda_{q^{r-\frac{1}{2}}}\left(\widetilde{\xi_{\mathbf{C}}}\right) \otimes \bigotimes_{s=1}^{\infty} \Lambda_{q^{s}}\left(\widetilde{\xi_{\mathbf{C}}}\right)
\end{aligned}
$$

Recall that for an indeterminate $t$,

$$
\Lambda_{t}(E)=\mathbf{C}\left|M+t E+t^{2} \Lambda^{2}(E)+\cdots, \quad S_{t}(E)=\mathbf{C}\right| M+t E+t^{2} S^{2}(E)+\cdots
$$


are respectively the total exterior and symmetric powers of $E$. The following relations hold between these two operations (cf. 2]),

$$
S_{t}(E)=\frac{1}{\Lambda_{-t}(E)}, \quad \Lambda_{t}(E-F)=\frac{\Lambda_{t}(E)}{\Lambda_{t}(F)} .
$$

We can formally expand these four elements into Fourier series in $q^{\frac{1}{2}}$ :

$$
\begin{gathered}
\Theta_{1}\left(T_{\mathbf{C}} M\right)=A_{0}\left(T_{\mathbf{C}} M\right)+A_{1}\left(T_{\mathbf{C}} M\right) q^{\frac{1}{2}}+\cdots, \\
\Theta_{2}\left(T_{\mathbf{C}} M\right)=B_{0}\left(T_{\mathbf{C}} M\right)+B_{1}\left(T_{\mathbf{C}} M\right) q^{\frac{1}{2}}+\cdots, \\
\Theta_{1}\left(T_{\mathbf{C}} M, \xi_{\mathbf{C}}\right)=A_{0}\left(T_{\mathbf{C}} M, \xi_{\mathbf{C}}\right)+A_{1}\left(T_{\mathbf{C}} M, \xi_{\mathbf{C}}\right) q^{\frac{1}{2}}+\cdots, \\
\Theta_{2}\left(T_{\mathbf{C}} M, \xi_{\mathbf{C}}\right)=B_{0}\left(T_{\mathbf{C}} M, \xi_{\mathbf{C}}\right)+B_{1}\left(T_{\mathbf{C}} M, \xi_{\mathbf{C}}\right) q^{\frac{1}{2}}+\cdots,
\end{gathered}
$$

where the $A_{i}$ 's and $B_{i}$ 's, are elements in the semi-group formally generated by Hermitian vector bundles over $M$. Moreover, they carry canonically induced Hermitian connections.

Let $c=e\left(\xi, \nabla^{\xi}\right)$ be the Euler form of $\xi$ canonically associated to $\nabla^{\xi}$.

Now we can state our twisted cancellation formulas and discuss their applications in the following subsections.

Theorem 2.1. For an $8 k+4$ dimensional smooth Riemannian manifold $M$, the following identity holds:

$$
\begin{gathered}
\left\{\widehat{L}\left(T M, \nabla^{T M}\right) \operatorname{ch}\left(T_{\mathbf{C}} M\right)-16 \widehat{L}\left(T M, \nabla^{T M}\right)\right\}^{(8 k+4)} \\
=2^{14}\left[\sum_{r=0}^{k-1}(k-r) 2^{6(k-r-1)} h_{r}\left(T_{\mathbf{C}} M\right)\right]
\end{gathered}
$$

where each $h_{r}\left(T_{\mathbf{C}} M\right)=\left\{\widehat{A}\left(T M, \nabla^{T M}\right) \operatorname{ch}\left(b_{r}\left(T_{\mathbf{C}} M\right)\right)\right\}^{(8 k+4)}, 0 \leq r \leq k$, and each $b_{r}\left(T_{\mathbf{C}} M\right)$ is a canonical integral linear combination of $B_{j}\left(T_{\mathbf{C}} M\right), 0 \leq j \leq r$. The right hand side is understood as 0 when $k<1$.

In Theorem 2.1, putting $k=0,1,2$, by computing $h_{0}\left(T_{\mathbf{C}} M\right)$ and $h_{1}\left(T_{\mathbf{C}} M\right)$ (see (5.18) and (5.19)), we have

Corollary 2.1. If $M$ is 4 dimensional, one has

$$
\left\{\widehat{L}\left(T M, \nabla^{T M}\right) \operatorname{ch}\left(T_{\mathbf{C}} M\right)-16 \widehat{L}\left(T M, \nabla^{T M}\right)\right\}^{(4)}=0 .
$$

Corollary 2.2. If $M$ is 12 dimensional, one has

$$
\left\{\widehat{L}\left(T M, \nabla^{T M}\right) \operatorname{ch}\left(T_{\mathbf{C}} M\right)-16 \widehat{L}\left(T M, \nabla^{T M}\right)\right\}^{(12)}=-2^{14}\left\{\widehat{A}\left(T M, \nabla^{T M}\right)\right\}^{(12)} .
$$

Corollary 2.3. If $M$ is 20 dimensional, one has

$$
\begin{gathered}
\left\{\widehat{L}\left(T M, \nabla^{T M}\right) \operatorname{ch}\left(T_{\mathbf{C}} M\right)-16 \widehat{L}\left(T M, \nabla^{T M}\right)\right\}^{(20)} \\
=2^{14}\left\{\widehat{A}\left(T M, \nabla^{T M}\right) \operatorname{ch}\left(T_{\mathbf{C}} M\right)-28 \widehat{A}\left(T M, \nabla^{T M}\right)\right\}^{(20)} .
\end{gathered}
$$


We also have

Theorem 2.2. For an $8 k+4$ dimensional smooth Riemannian manifold $M$, the following identity holds:

$$
\begin{gathered}
\left\{\widehat{L}\left(T M, \nabla^{T M}\right) \operatorname{ch}\left(T_{\mathbf{C}} M \otimes T_{\mathbf{C}} M\right)-\right. \\
+55 \widehat{L}\left(T M, \nabla^{T M}\right) \operatorname{ch}\left(T_{\mathbf{C}} M\right) \\
\left.+768 \widehat{L}\left(T M, \nabla^{T M}\right)\right\}^{(8 k+4)} \\
=2^{25}\left[\sum_{r=0}^{k-2}(k-r)(k-r-1) 2^{6(k-r-2)} h_{r}\left(T_{\mathbf{C}} M\right)\right],
\end{gathered}
$$

where each $h_{r}\left(T_{\mathbf{C}} M\right)=\left\{\widehat{A}\left(T M, \nabla^{T M}\right) \operatorname{ch}\left(b_{r}\left(T_{\mathbf{C}} M\right)\right)\right\}^{(8 k+4)}, 0 \leq r \leq k$, and each $b_{r}\left(T_{\mathbf{C}} M\right)$ is a canonical integral linear combination of $B_{j}\left(T_{\mathbf{C}} M\right), 0 \leq j \leq r$. The right hand side is understood as 0 when $k<2$.

In Theorem 2.2, putting $k=0,1,2,3$, by computing $h_{0}\left(T_{\mathbf{C}} M\right)$ and $h_{1}\left(T_{\mathbf{C}} M\right)$ (see (5.18) and (5.19)), we have

Corollary 2.4. If $M$ is 4 dimensional, one has

$$
\begin{aligned}
\left\{\widehat{L}\left(T M, \nabla^{T M}\right) \operatorname{ch}\left(T_{\mathbf{C}} M \otimes T_{\mathbf{C}} M\right)-55 \widehat{L}(T M,\right. & \left.\nabla^{T M}\right) \operatorname{ch}\left(T_{\mathbf{C}} M\right) \\
& \left.+768 \widehat{L}\left(T M, \nabla^{T M}\right)\right\}^{(4)}=0 .
\end{aligned}
$$

Equivalently, in view of Corollary 2.1, one has

$$
\left\{\widehat{L}\left(T M, \nabla^{T M}\right) \operatorname{ch}\left(T_{\mathbf{C}} M \otimes T_{\mathbf{C}} M\right)-112 \widehat{L}\left(T M, \nabla^{T M}\right)\right\}^{(4)}=0 .
$$

Corollary 2.5. If $M$ is 12 dimensional, one has

$$
\begin{aligned}
\left\{\widehat{L}\left(T M, \nabla^{T M}\right) \operatorname{ch}\left(T_{\mathbf{C}} M \otimes T_{\mathbf{C}} M\right)-55 \widehat{L}\left(T M, \nabla^{T M}\right)\right. & \operatorname{ch}\left(T_{\mathbf{C}} M\right) \\
& \left.+768 \widehat{L}\left(T M, \nabla^{T M}\right)\right\}^{(12)}=0 .
\end{aligned}
$$

Corollary 2.6. If $M$ is 20 dimensional, one has

$$
\begin{aligned}
\left\{\widehat{L}\left(T M, \nabla^{T M}\right) \operatorname{ch}\left(T_{\mathbf{C}} M \otimes T_{\mathbf{C}} M\right)-\right. & 55 \widehat{L}\left(T M, \nabla^{T M}\right) \operatorname{ch}\left(T_{\mathbf{C}} M\right) \\
+ & \left.768 \widehat{L}\left(T M, \nabla^{T M}\right)\right\}^{(20)} \\
& =-2^{26}\left\{\widehat{A}\left(T M, \nabla^{T M}\right)\right\}^{(20)} .
\end{aligned}
$$

Corollary 2.7. If $M$ is 28 dimensional, one has

$$
\begin{aligned}
&\left\{\widehat{L}\left(T M, \nabla^{T M}\right) \operatorname{ch}\left(T_{\mathbf{C}} M \otimes T_{\mathbf{C}} M\right)-\right. 55 \widehat{L}\left(T M, \nabla^{T M}\right) \operatorname{ch}\left(T_{\mathbf{C}} M\right) \\
&\left.+768 \widehat{L}\left(T M, \nabla^{T M}\right)\right\}^{(28)} \\
&=2^{26}\left\{\widehat{A}\left(T M, \nabla^{T M}\right) \operatorname{ch}\left(T_{\mathbf{C}} M\right)-52 \widehat{A}\left(T M, \nabla^{T M}\right)\right\}^{(28)}
\end{aligned}
$$


For $8 k$ dimensional manifolds, we have the following twisted cancellation formulas.

Theorem 2.3. For an $8 k$ dimensional smooth Riemannian manifold $M$, the following identity holds:

$$
\left\{\widehat{L}\left(T M, \nabla^{T M}\right) \operatorname{ch}\left(T_{\mathbf{C}} M\right)\right\}^{(8 k)}=2^{11}\left[\sum_{r=0}^{k-1}(k-r) 2^{6(k-r-1)} z_{r}\left(T_{\mathbf{C}} M\right)\right],
$$

where each $z_{r}\left(T_{\mathbf{C}} M\right)=\left\{\widehat{A}\left(T M, \nabla^{T M}\right) \operatorname{ch}\left(d_{r}\left(T_{\mathbf{C}} M\right)\right)\right\}^{(8 k)}, 0 \leq r \leq k$, and each $d_{r}\left(T_{\mathbf{C}} M\right)$ is a canonical integral linear combination of $B_{j}\left(T_{\mathbf{C}} M\right), 0 \leq j \leq r$.

In Theorem 2.3, putting $k=1,2$, by computing $z_{0}\left(T_{\mathbf{C}} M\right)$ and $z_{1}\left(T_{\mathbf{C}} M\right)$ (see (5.32) and (5.33)), we have

Corollary 2.8. If $M$ is 8 dimensional, one has

$$
\left\{\widehat{L}\left(T M, \nabla^{T_{\mathbf{C}} M}\right) \operatorname{ch}\left(T_{\mathbf{C}} M\right)\right\}^{(8)}=2048\left\{\widehat{A}\left(T M, \nabla^{T M}\right)\right\}^{(8)} .
$$

Corollary 2.9. If $M$ is 16 dimensional, one has

$$
\begin{aligned}
\left\{\widehat{L}\left(T M, \nabla^{T M}\right) \operatorname{ch}\left(T_{\mathbf{C}} M\right)\right\}^{(16)} & \\
= & -2048\left\{\widehat{A}\left(T M, \nabla^{T M}\right) \operatorname{ch}\left(T_{\mathbf{C}} M\right)-48 \widehat{A}\left(T M, \nabla^{T M}\right)\right\}^{(16)} .
\end{aligned}
$$

We also have

Theorem 2.4. For an $8 k$ dimensional smooth Riemannian manifold $M$, the following identity holds:

$$
\begin{aligned}
& \left\{\widehat{L}\left(T M, \nabla^{T M}\right) \operatorname{ch}\left(T_{\mathbf{C}} M \otimes T_{\mathbf{C}} M\right)-23 \widehat{L}\left(T M, \nabla^{T M}\right) \operatorname{ch}\left(T_{\mathbf{C}} M\right)\right\}^{(8 k)} \\
= & 2^{22}\left[\sum_{r=0}^{k-2}(k-r)(k-r-1) 2^{6(k-r-2)} z_{r}\left(T_{\mathbf{C}} M\right)\right],
\end{aligned}
$$

where each $z_{r}\left(T_{\mathbf{C}} M\right)=\left\{\widehat{A}\left(T M, \nabla^{T M}\right) \operatorname{ch}\left(d_{r}\left(T_{\mathbf{C}} M\right)\right)\right\}^{(8 k)}, 0 \leq r \leq k$, and each $d_{r}\left(T_{\mathbf{C}} M\right)$ is a canonical integral linear combination of $B_{j}\left(T_{\mathbf{C}} M\right), 0 \leq j \leq r$. The right hand side is understood as 0 when $k<2$.

In Theorem 2.4, putting $k=1,2,3$, by computing $z_{0}\left(T_{\mathbf{C}} M\right)$ and $z_{1}\left(T_{\mathbf{C}} M\right)$ (see (5.32) and (5.33)), we have

Corollary 2.10. If $M$ is 8 dimensional, one has

$$
\left\{\widehat{L}\left(T M, \nabla^{T M}\right) \operatorname{ch}\left(T_{\mathbf{C}} M \otimes T_{\mathbf{C}} M\right)-23 \widehat{L}\left(T M, \nabla^{T M}\right) \operatorname{ch}\left(T_{\mathbf{C}} M\right)\right\}^{(8)}=0 .
$$

Equivalently, in view of Corollary 2.8, one has

$$
\left\{\widehat{L}\left(T M, \nabla^{T M}\right) \operatorname{ch}\left(T_{\mathbf{C}} M \otimes T_{\mathbf{C}} M\right)\right\}^{(8)}=\left\{23 \cdot 2048 \widehat{A}\left(T M, \nabla^{T M}\right)\right\}^{(8)} .
$$


Corollary 2.11. If $M$ is 16 dimensional, one has

$$
\begin{aligned}
\left\{\widehat{L}\left(T M, \nabla^{T M}\right) \operatorname{ch}\left(T_{\mathbf{C}} M \otimes T_{\mathbf{C}} M\right)-23 \widehat{L}(T M,\right. & \left.\left.\nabla^{T M}\right) \operatorname{ch}\left(T_{\mathbf{C}} M\right)\right\}^{(16)} \\
& =2^{23}\left\{\widehat{A}\left(T M, \nabla^{T M}\right)\right\}^{(16)} .
\end{aligned}
$$

Corollary 2.12. If $M$ is 24 dimensional, one has

$$
\begin{aligned}
& \left\{\widehat{L}\left(T M, \nabla^{T M}\right) \operatorname{ch}\left(T_{\mathbf{C}} M \otimes T_{\mathbf{C}} M\right)-23 \widehat{L}\left(T M, \nabla^{T M}\right) \operatorname{ch}\left(T_{\mathbf{C}} M\right)\right\}^{(24)} \\
= & -2^{23}\left\{\widehat{A}\left(T M, \nabla^{T M}\right) \operatorname{ch}\left(T_{\mathbf{C}} M\right)-72 \widehat{A}\left(T M, \nabla^{T M}\right)\right\}^{(24)} .
\end{aligned}
$$

If we include the extra complex line bundle $\xi$ into our picture, we have the following.

Theorem 2.5. For an $8 k+4$ dimensional smooth Riemannian manifold $M$, the following identity holds:

$$
\begin{gathered}
\left\{\frac{\widehat{L}\left(T M, \nabla^{T M}\right)\left[\operatorname{ch}\left(T_{\mathbf{C}} M, \nabla^{T_{\mathbf{C}} M}\right)-\sinh ^{2}\left(\frac{c}{2}\right) \operatorname{ch}\left(2 \xi_{\mathbf{C}} \oplus \mathbf{C}^{8}\right)-16\right]}{\cosh ^{2}\left(\frac{c}{2}\right)}\right\}^{(8 k+4)} \\
=2^{14}\left[\sum_{r=0}^{k-1}(k-r) 2^{6(k-r-1)} h_{r}\left(T_{\mathbf{C}} M, \xi_{\mathbf{C}}\right)\right]
\end{gathered}
$$

where each $h_{r}\left(T_{\mathbf{C}} M, \xi_{\mathbf{C}}\right)=\left\{\widehat{A}\left(T M, \nabla^{T M}\right) \operatorname{ch}\left(b_{r}\left(T_{\mathbf{C}} M, \xi_{\mathbf{C}}\right)\right) \cosh \left(\frac{c}{2}\right)\right\}^{(8 k+4)}, 0 \leq r \leq$ $k$, and each $b_{r}\left(T_{\mathbf{C}} M, \xi_{\mathbf{C}}\right)$ is a canonical integral linear combination of $B_{j}\left(T_{\mathbf{C}} M, \xi_{\mathbf{C}}\right)$, $0 \leq j \leq r$. The right hand is understood as 0 when $k<1$.

For the $8 k$ dimensional case, we have the following.

Theorem 2.6. For an $8 k$ dimensional smooth Riemannian manifold $M$, the following identity holds:

$$
\begin{gathered}
\left\{\frac{\widehat{L}\left(T M, \nabla^{T M}\right)\left[\operatorname{ch}\left(T_{\mathbf{C}} M, \nabla^{T_{\mathbf{C}} M}\right)-\sinh ^{2}\left(\frac{c}{2}\right) \operatorname{ch}\left(2 \xi_{\mathbf{C}} \oplus \mathbf{C}^{8}\right)\right]}{\cosh ^{2}\left(\frac{c}{2}\right)}\right\}^{(8 k)} \\
=2^{11}\left[\sum_{r=0}^{k-1}(k-r) 2^{6(k-r-1)} z_{r}\left(T_{\mathbf{C}} M, \xi_{\mathbf{C}}\right)\right]
\end{gathered}
$$

where each $z_{r}\left(T_{\mathbf{C}} M, \xi_{\mathbf{C}}\right)=\left\{\widehat{A}\left(T M, \nabla^{T M}\right) \operatorname{ch}\left(d_{r}\left(T_{\mathbf{C}} M, \xi_{\mathbf{C}}\right)\right) \cosh \left(\frac{c}{2}\right)\right\}^{(8 k)}, 0 \leq r \leq k$, and each $d_{r}\left(T_{\mathbf{C}} M, \xi_{\mathbf{C}}\right)$ is a canonical integral linear combination of $B_{j}\left(T_{\mathbf{C}} M, \xi_{\mathbf{C}}\right)$, $0 \leq j \leq r$.

\section{Spin manifolds AND Divisibilities of twisted Signatures}

Let $\operatorname{Sig}(M, T)$ and $\operatorname{Sig}(M, T \otimes T)$ denote $\operatorname{Sig}\left(M, T_{\mathbf{C}} M\right)$ and $\operatorname{Sig}\left(M, T_{\mathbf{C}} M \otimes T_{\mathbf{C}} M\right)$ respectively. In this section, we apply Theorems $2.1,2.2$ and Theorems $2.3,2.4$ to $8 k+4$ and $8 k$ dimensional closed spin manifolds respectively to obtain divisibility results for the twisted signatures $\operatorname{Sig}(M, T)$ and $\operatorname{Sig}(M, T \otimes T)$. We also show that our divisibilities are best possible. 
3.1. $8 k+4$ dimensional case. According to the generalized Hirzebruch signature formula [7, 11, when $M$ is an $8 k+4$ dimensional closed spin manifold, integrating both sides of (2.13) against the fundamental class $[M]$, we have

$$
\operatorname{Sig}(M, T)-16 \operatorname{Sig}(M)=2^{14}\left\langle\left[\sum_{r=0}^{k-1}(k-r) 2^{6(k-r-1)} h_{r}\left(T_{\mathbf{C}} M\right)\right],[M]\right\rangle .
$$

According to the Atiyah-Hirzebruch divisibility [3, we obtain

Theorem 3.1. If $M$ is an $8 k+4$ dimensional closed spin manifold, then $(\operatorname{Sig}(M, T)-16 \operatorname{Sig}(M))$ is divisible by $2^{15}$.

Then according to Theorem 3.1 and the Ochanine divisibility [19 that the signature of $8 k+4$ dimensional closed spin manifolds is divisible by 16 , we see that our twisted cancellation formula (2.13) actually implies the Hirzebruch divisibility:

Theorem 3.2 (Hirzebruch, [12). If $M$ is an $8 k+4$ dimensional closed spin manifold, then the twisted signature $\operatorname{Sig}(M, T)$ is divisible by 256 .

Moreover, we are able to show that the Hirzebruch divisibility is best possible.

Proposition 3.1. 256 is the best possible divisibility of the twisted signature $\operatorname{Sig}(M, T)$ for $8 k+4$ dimensional spin manifolds.

To prove Proposition 3.1, we need the following lemmas, which are easy to deduce directly.

Lemma 3.1. Let $M_{1}$ and $M_{2}$ be two closed oriented smooth manifolds. Then one has

$$
\operatorname{Sig}\left(M_{1} \times M_{2}, T\right)=\operatorname{Sig}\left(M_{1}\right) \operatorname{Sig}\left(M_{2}, T\right)+\operatorname{Sig}\left(M_{2}\right) \operatorname{Sig}\left(M_{1}, T\right),
$$

and

$$
\begin{aligned}
& \operatorname{Sig}\left(M_{1} \times M_{2}, T \otimes T\right) \\
= & \operatorname{Sig}\left(M_{1}\right) \operatorname{Sig}\left(M_{2}, T \otimes T\right)+2 \operatorname{Sig}\left(M_{1}, T\right) \operatorname{Sig}\left(M_{2}, T\right)+\operatorname{Sig}\left(M_{2}\right) \operatorname{Sig}\left(M_{1}, T \otimes T\right) .
\end{aligned}
$$

Proof. Let $p_{1}: M_{1} \times M_{2} \rightarrow M_{1}, p_{2}: M_{1} \times M_{2} \rightarrow M_{2}$ be the two projections. It's not hard to see that

$$
\begin{aligned}
& \int_{M_{1} \times M_{2}} \widehat{L}\left(M_{1} \times M_{2}\right) \operatorname{ch}\left(T_{C}\left(M_{1} \times M_{2}\right)\right) \\
= & \int_{M_{1} \times M_{2}} \widehat{L}\left(p_{1}^{*}\left(T M_{1}\right)\right) \widehat{L}\left(p_{2}^{*}\left(T M_{2}\right)\right)\left(\operatorname{ch}\left(p_{1}^{*}\left(T_{\mathbf{C}} M_{1}\right)\right)+\operatorname{ch}\left(p_{2}^{*}\left(T_{\mathbf{C}} M_{2}\right)\right)\right) \\
= & \int_{M_{1} \times M_{2}} p_{1}^{*}\left(\widehat{L}\left(M_{1}\right)\right) p_{2}^{*}\left(\widehat{L}\left(M_{2}\right) \operatorname{ch}\left(T_{\mathbf{C}} M_{2}\right)\right)+p_{2}^{*}\left(\widehat{L}\left(M_{2}\right)\right) p_{1}^{*}\left(\widehat{L}\left(M_{1}\right) \operatorname{ch}\left(T_{\mathbf{C}} M_{1}\right)\right) \\
= & \int_{M_{1}} \widehat{L}\left(M_{1}\right) \int_{M_{2}} \widehat{L}\left(M_{2}\right) \operatorname{ch}\left(T_{\mathbf{C}} M_{2}\right)+\int_{M_{2}} \widehat{L}\left(M_{2}\right) \int_{M_{1}} \widehat{L}\left(M_{1}\right) \operatorname{ch}\left(T_{\mathbf{C}} M_{1}\right) .
\end{aligned}
$$

Thus we have

$$
\operatorname{Sig}\left(M_{1} \times M_{2}, T\right)=\operatorname{Sig}\left(M_{1}\right) \operatorname{Sig}\left(M_{2}, T\right)+\operatorname{Sig}\left(M_{2}\right) \operatorname{Sig}\left(M_{1}, T\right) .
$$

By similar computations, it's not hard to prove (3.3). 
Lemma 3.2. Let $\mathbf{H} P^{2}$ be the quaternionic projective plane. Then for the $8 n$ dimensional manifold $\left(\mathbf{H} P^{2}\right)^{n}$, the $n$-fold product of $\mathbf{H} P^{2}$, one has $\operatorname{Sig}\left(\left(\mathbf{H} P^{2}\right)^{n}\right)=1$, $\operatorname{Sig}\left(\left(\mathbf{H} P^{2}\right)^{n}, T\right)=0$ and $\operatorname{Sig}\left(\left(\mathbf{H} P^{2}\right)^{n}, T \otimes T\right)=0$, where $n$ is a positive integer.

Proof. Let $\mathbf{H} P^{n}$ be the quaternionic projective space and $u$ be the generator of $H^{4}\left(\mathbf{H} P^{n}, \mathbf{Z}\right)$. A theorem of Hirzebruch ([10]) says that the total Pontrjagin class of $T \mathbf{H} P^{n}$ is the following:

$$
p\left(\mathbf{H} P^{n}\right)=(1+u)^{2 n+2}(1+4 u)^{-1}=(1+u)^{2 n+2}\left(1-4 u+16 u^{2}+\cdots\right) .
$$

In particular, for $\mathbf{H} P^{2}$, we have $p_{1}\left(\mathbf{H} P^{2}\right)=2 u, p_{2}\left(\mathbf{H} P^{2}\right)=7 u^{2}$.

By direct computations, $\operatorname{Sig}\left(\mathbf{H} P^{2}\right)=\left\langle-\frac{1}{45} p_{1}^{2}+\frac{7}{45} p_{2},\left[\mathbf{H} P^{2}\right]\right\rangle=1$. Thus by the multiplicity of the signature, we have $\operatorname{Sig}\left(\left(\mathbf{H} P^{2}\right)^{n}\right)=1$, for $n \in \mathbf{Z}^{+}$.

Also by direct computations, we have $\widehat{A}\left(\mathbf{H} P^{2}\right)=\left\langle\frac{1}{16}\left(\frac{7}{360} p_{1}^{2}-\frac{1}{90} p_{2}\right),\left[\mathbf{H} P^{2}\right]\right\rangle=0$. Thus by Corollary 2.8, we have $\operatorname{Sig}\left(\mathbf{H} P^{2}, T\right)=0$. Continuing to apply (3.2), we obtain that $\operatorname{Sig}\left(\left(\mathbf{H} P^{2}\right)^{n}, T\right)=0$, for $n \in \mathbf{Z}^{+}$.

By Corollary 2.10, $\operatorname{Sig}\left(\mathbf{H} P^{2}, T \otimes T\right)=23 \operatorname{Sig}\left(\mathbf{H} P^{2}, T\right)=0$. Continuing to apply (3.3), we obtain that $\operatorname{Sig}\left(\left(\mathbf{H} P^{2}\right)^{n}, T \otimes T\right)=0$, for $n \in \mathbf{Z}^{+}$.

Now we can prove Proposition 3.1 as follows.

Proof. Let $K$ be a K3-surface. It is well known that $\operatorname{Sig}(K)$ is -16 . Then by Corollary 2.1, $\operatorname{Sig}(K, T)=16 \operatorname{Sig}(K)=-256$.

Applying (3.2) and Lemma 3.2 to the $8 k+4$ dimensional spin manifold $K \times$ $\left(\mathbf{H} P^{2}\right)^{k}$, we obtain that

$$
\begin{aligned}
& \operatorname{Sig}\left(K \times\left(\mathbf{H} P^{2}\right)^{k}, T\right) \\
= & \operatorname{Sig}(K) \operatorname{Sig}\left(\left(\mathbf{H} P^{2}\right)^{k}, T\right)+\operatorname{Sig}\left(\left(\mathbf{H} P^{2}\right)^{k}\right) \operatorname{Sig}(K, T) \\
= & \operatorname{Sig}(K, T) \\
= & -256 .
\end{aligned}
$$

This proves Proposition 3.1.

Our twisted cancellation formula (2.17) implies the divisibility of the twisted signature $\operatorname{Sig}(M, T \otimes T)$. According to the generalized Hirzebruch signature formula [7, 11, when $M$ is an $8 k+4$ dimensional closed spin manifold, integrating both sides of (2.17) against the fundamental class $[M]$, we have

$$
\begin{aligned}
& \operatorname{Sig}(M, T \otimes T)-55 \operatorname{Sig}(M, T)+768 \operatorname{Sig}(M) \\
= & 2^{25}\left\langle\left[\sum_{r=0}^{k-2}(k-r)(k-r-1) 2^{6(k-r-2)} h_{r}\left(T_{\mathbf{C}} M\right)\right],[M]\right\rangle .
\end{aligned}
$$

In particular, by (2.19), in dimension 4, we have

$$
\operatorname{Sig}(M, T \otimes T)-112 \operatorname{Sig}(M)=0 .
$$

By the Atiyah-Hirzebruch divisibility [3], (3.5) shows that $\operatorname{Sig}(M, T \otimes T)$ $-55 \operatorname{Sig}(M, T)+768 \operatorname{Sig}(M)$ is divisible by $2^{26}$. Therefore, according to Theorem 3.2 and the Ochanine divisibility [19], we obtain

Theorem 3.3. Let $M$ be an $8 k+4$ dimensional closed spin manifold. When $\operatorname{dim} M=4$, the twisted signature $\operatorname{Sig}(M, T \otimes T)$ is divisible by $256 \cdot 7 ;$ when $\operatorname{dim} M=$ $8 k+4, k \geq 1$, the the twisted signature $\operatorname{Sig}(M, T \otimes T)$ is divisible by 256 . 
Moreover we are also able to show that these divisibilities of $\operatorname{Sig}(M, T \otimes T)$ are best possible.

Proposition 3.2. $256 \cdot 7$ is the best possible divisibility of the twisted signature $\operatorname{Sig}(M, T \otimes T)$ for 4 dimensional spin manifolds; 256 is the best possible divisibility of the twisted signature $\operatorname{Sig}(M, T \otimes T)$ for $8 k+4$ dimensional spin manifolds, where $k \geq 1$.

Proof. Let $K$ be a K3-surface. By (3.6), one has $\operatorname{Sig}(K, T \otimes T)=112 \operatorname{Sig}(K)=$ $-256 \cdot 7$. This shows $256 \cdot 7$ is the best possible divisibility of the twisted signature $\operatorname{Sig}(M, T \otimes T)$ for 4 dimensional spin manifolds.

Let $B^{8}$ be such a Bott manifold, which is 8 dimensional, spin with $\widehat{A}\left(B^{8}\right)=1$ and $\operatorname{Sig}\left(B^{8}\right)=0$ [15]. By Corollary 2.8, $\operatorname{Sig}\left(B^{8}, T\right)=2048 \widehat{A}\left(B^{8}\right)=2048$. Then by (2.27), $\operatorname{Sig}\left(B^{8}, T \otimes T\right)=23 \operatorname{Sig}\left(B^{8}, T\right)=23 \cdot 2048$. Therefore, by (3.3),

$$
\begin{aligned}
& \operatorname{Sig}\left(K \times B^{8}, T \otimes T\right) \\
= & \operatorname{Sig}(K) \operatorname{Sig}\left(B^{8}, T \otimes T\right)+2 \operatorname{Sig}(K, T) \operatorname{Sig}\left(B^{8}, T\right)+\operatorname{Sig}\left(B^{8}\right) \operatorname{Sig}(K, T \otimes T) \\
= & -16 \cdot 23 \cdot 2048+2 \cdot(-256) \cdot 2048 \\
= & -55 \cdot 2^{15} .
\end{aligned}
$$

Applying (3.3) and Lemma 3.2 to the $8 k+4$ dimensional, $k \geq 1$, spin manifold $\left(\mathbf{H} P^{2}\right)^{k-1} \times K \times B^{8}$, we have

$$
\begin{aligned}
& \operatorname{Sig}\left(\left(\mathbf{H} P^{2}\right)^{k-1} \times K \times B^{8}, T \otimes T\right) \\
= & \operatorname{Sig}\left(K \times B^{8}\right) \operatorname{Sig}\left(\left(\mathbf{H} P^{2}\right)^{k-1}, T \otimes T\right)+2 \operatorname{Sig}\left(K \times B^{8}, T\right) \operatorname{Sig}\left(\left(\mathbf{H} P^{2}\right)^{k-1}, T\right) \\
& +\operatorname{Sig}\left(\left(\mathbf{H} P^{2}\right)^{k-1}\right) \operatorname{Sig}\left(K \times B^{8}, T \otimes T\right) \\
= & \operatorname{Sig}\left(K \times B^{8}, T \otimes T\right) \\
= & -55 \cdot 2^{15} .
\end{aligned}
$$

Applying (3.3) and Lemma 3.2 to the $8 k+4$ dimensional, $k \geq 1$, spin manifold $\left(\mathbf{H} P^{2}\right)^{k} \times K$, we have

$$
\begin{aligned}
& \operatorname{Sig}\left(\left(\mathbf{H} P^{2}\right)^{k} \times K, T \otimes T\right) \\
= & \operatorname{Sig}(K) \operatorname{Sig}\left(\left(\mathbf{H} P^{2}\right)^{k}, T \otimes T\right)+2 \operatorname{Sig}(K, T) \operatorname{Sig}\left(\left(\mathbf{H} P^{2}\right)^{k}, T\right) \\
& +\operatorname{Sig}\left(\left(\mathbf{H} P^{2}\right)^{k}\right) \operatorname{Sig}(K, T \otimes T) \\
= & \operatorname{Sig}(K, T \otimes T) \\
= & -256 \cdot 7 .
\end{aligned}
$$

Since the maximal common denominator of $-55 \cdot 2^{15}$ and $-256 \cdot 7$ is 256 , we see that 256 is the best possible divisibility of the twisted signature $\operatorname{Sig}(M, T \otimes T)$ for $8 k+4$ dimensional spin manifolds, where $k \geq 1$.

3.2. $8 k$ dimensional case. According to the generalized Hirzebruch signature formula [7, when $M$ is an $8 k$ dimensional closed spin manifold, integrating both sides of (2.23) against the fundamental class $[M]$, we have

$$
\operatorname{Sig}(M, T)=2048\left\langle\left[\sum_{r=0}^{k-1}(k-r) 2^{6(k-r-1)} z_{r}\left(T_{\mathbf{C}} M\right)\right],[M]\right\rangle .
$$


Then according to the Atiyah-Singer index theorem, our anomaly cancellation formula (2.23) actually implies the Hirzebruch divisibility:

Theorem 3.4 (Hirzebruch, [12]). If $M$ is an $8 k$ dimensional closed spin manifold, then $\operatorname{Sig}(M, T)$ is divisible by 2048 .

Remark 3.1. This divisibility looks astonishing since on $8 k$ dimensional closed spin manifolds, we can say nothing about the divisibility on the signature while this twisted signature has such high divisibility.

Moreover, we are able to show that the Hirzebruch divisibility is best possible.

Proposition 3.3. 2048 is the best possible divisibility for the tangent twisted signature of $8 k$ dimensional spin manifolds.

Proof. Let $B^{8}$ be a Bott manifold as in the proof of Proposition 3.2. Applying Lemma 3.1 and Lemma 3.2 to the $8 k$ dimensional spin manifold $B^{8} \times\left(\mathbf{H} P^{2}\right)^{k-1}$, one has

$$
\begin{aligned}
& \operatorname{Sig}\left(B^{8} \times\left(\mathbf{H} P^{2}\right)^{k-1}, T\right) \\
= & \operatorname{Sig}\left(B^{8}\right) \operatorname{Sig}\left(\left(\mathbf{H} P^{2}\right)^{k-1}, T\right)+\operatorname{Sig}\left(\left(\mathbf{H} P^{2}\right)^{k-1}\right) \operatorname{Sig}\left(B^{8}, T\right) \\
= & \operatorname{Sig}\left(B^{8}, T\right) \\
= & 2048 .
\end{aligned}
$$

This proves Proposition 3.3.

Our twisted cancellation formula (2.26) implies the divisibility of the twisted signature $\operatorname{Sig}(M, T \otimes T)$. According to the generalized Hirzebruch signature formula [7, 11, when $M$ is an $8 k$ dimensional closed spin manifold, integrating both sides of (2.26) against the fundamental class $[M]$, we have

$$
\begin{aligned}
& \operatorname{Sig}(M, T \otimes T)-23 \operatorname{Sig}(M, T) \\
= & 2^{22}\left\langle\left[\sum_{r=0}^{k-2}(k-r)(k-r-1) 2^{6(k-r-2)} z_{r}\left(T_{\mathbf{C}} M\right)\right],[M]\right\rangle .
\end{aligned}
$$

In particular, by (2.28), in dimension 8 , we have

$$
\operatorname{Sig}(M, T \otimes T)-23 \cdot 2048 \widehat{A}(M)=0 .
$$

By the Atiyah-Singer index theorem, $\operatorname{Sig}(M, T \otimes T)-23 \operatorname{Sig}(M, T)$ is divisible by $2^{22}$. Therefore, according to Theorem 3.4, we obtain

Theorem 3.5. Let $M$ be an $8 k$ dimensional closed spin manifold. When $\operatorname{dim} M=$ 8 , the twisted signature $\operatorname{Sig}(M, T \otimes T)$ is divisible by $2048 \cdot 23$; when $\operatorname{dim} M=8 k, k \geq$ 2 , the twisted signature $\operatorname{Sig}(M, T \otimes T)$ is divisible by 2048 .

Moreover, we are also able to show that these divisibilities of $\operatorname{Sig}(M, T \otimes T)$ are best possible.

Proposition 3.4. $2048 \cdot 23$ is the best possible divisibility of the twisted signature $\operatorname{Sig}(M, T \otimes T)$ for 8 dimensional spin manifolds; 2048 is the best possible divisibility of the twisted signature $\operatorname{Sig}(M, T \otimes T)$ for $8 k$ dimensional spin manifolds, where $k \geq 2$. 
Proof. Let $B^{8}$ be a Bott manifold as in the proof of Proposition 3.2. Then $\operatorname{Sig}\left(B^{8}, T \otimes T\right)=2048 \cdot 23 \widehat{A}\left(B^{8}\right)=2048 \cdot 23$ shows that $2048 \cdot 23$ is the best possible divisibility of the twisted signature $\operatorname{Sig}(M, T \otimes T)$ for 8 dimensional spin manifolds.

By (3.3),

$$
\begin{aligned}
& \operatorname{Sig}\left(B^{8} \times B^{8}, T \otimes T\right) \\
= & \operatorname{Sig}\left(B^{8}\right) \operatorname{Sig}\left(B^{8}, T \otimes T\right)+2 \operatorname{Sig}\left(B^{8}, T\right) \operatorname{Sig}\left(B^{8}, T\right)+\operatorname{Sig}\left(B^{8}\right) \operatorname{Sig}\left(B^{8}, T \otimes T\right) \\
= & 2 \operatorname{Sig}\left(B^{8}, T\right) \operatorname{Sig}\left(B^{8}, T\right) \\
= & 2^{23} .
\end{aligned}
$$

Applying (3.3) and Lemma 3.2 to the $8 k$ dimensional, $k \geq 2$, spin manifold $\left(\mathbf{H} P^{2}\right)^{k-2} \times B^{8} \times B^{8}$, we have

$$
\begin{aligned}
& \operatorname{Sig}\left(\left(\mathbf{H} P^{2}\right)^{k-2} \times B^{8} \times B^{8}, T \otimes T\right) \\
= & \operatorname{Sig}\left(B^{8} \times B^{8}\right) \operatorname{Sig}\left(\left(\mathbf{H} P^{2}\right)^{k-2}, T \otimes T\right)+2 \operatorname{Sig}\left(B^{8} \times B^{8}, T\right) \operatorname{Sig}\left(\left(\mathbf{H} P^{2}\right)^{k-2}, T\right) \\
& +\operatorname{Sig}\left(\left(\mathbf{H} P^{2}\right)^{k-2}\right) \operatorname{Sig}\left(B^{8} \times B^{8}, T \otimes T\right) \\
= & \operatorname{Sig}\left(B^{8} \times B^{8}, T \otimes T\right) \\
= & 2^{23} .
\end{aligned}
$$

Applying (3.3) and Lemma 3.2 to the $8 k$ dimensional, $k \geq 2$, spin manifold $\left(\mathbf{H} P^{2}\right)^{k-1} \times B^{8}$, we have

$$
\begin{aligned}
& \operatorname{Sig}\left(\left(\mathbf{H} P^{2}\right)^{k-1} \times B^{8}, T \otimes T\right) \\
= & \operatorname{Sig}\left(B^{8}\right) \operatorname{Sig}\left(\left(\mathbf{H} P^{2}\right)^{k-1}, T \otimes T\right)+2 \operatorname{Sig}\left(B^{8}, T\right) \operatorname{Sig}\left(\left(\mathbf{H} P^{2}\right)^{k-1}, T\right) \\
& +\operatorname{Sig}\left(\left(\mathbf{H} P^{2}\right)^{k-1}\right) \operatorname{Sig}\left(B^{8}, T \otimes T\right) \\
= & \operatorname{Sig}\left(B^{8}, T \otimes T\right) \\
= & 2048 \cdot 23 .
\end{aligned}
$$

Since the maximal common denominator of $2^{23}$ and $2048 \cdot 23$ is 2048 , we see that 2048 is the best possible divisibility of the twisted signature $\operatorname{Sig}(M, T \otimes T)$ for $8 k$ dimensional spin manifolds, where $k \geq 2$.

\section{Spin ${ }^{c}$ MANifolds AND TWisted ROKhlin CONGRUEnCES FOR CHARACTERISTIC NUMBERS}

In this section, we apply Theorem 2.5 and Theorem 2.6 to $8 k+4$ and $8 k$ dimensional $\operatorname{spin}^{c}$ manifolds respectively to obtain some congruence results. In particular, for $8 k+4$ dimensional $\operatorname{spin}^{c}$ manifolds, we establish twisted Rokhlin congruence formulas.

4.1. $8 k+4$ dimensional case. Let $M$ be an $8 k+4$ dimensional closed $\operatorname{spin}^{c}$ manifold and $\left(\xi, \nabla^{\xi}\right)$ be a real oriented Euclidean plane bundle, or equivalently a complex line bundle, over $M$ such that $w_{2}(T M) \equiv\left[e\left(\xi, \nabla^{\xi}\right)\right]$ in $H^{2}\left(M, \mathbf{Z}_{2}\right)$. Let $B$ be an oriented $8 k+2$ dimensional submanifold of $M$ such that $[B] \in H_{8 k+2}(M, \mathbf{Z})$ is dual to $\left[e\left(\xi, \nabla^{\xi}\right)\right]$. Let $B \bullet B$ denote the self-intersection of $B$ in $M$ and $N$ be 
the normal bundle to $B \bullet B$ in $M$. Applying the Poincaré duality, we have

$$
\begin{aligned}
& \int_{M} \frac{\widehat{L}(T M)}{\cosh ^{2}\left(\frac{c}{2}\right)}=\int_{M} \widehat{L}(T M)-\int_{M} \widehat{L}(T M) \frac{\sinh ^{2}\left(\frac{c}{2}\right)}{\cosh ^{2}\left(\frac{c}{2}\right)} \\
& =\int_{M} \widehat{L}(T M)-\int_{B \bullet B} \widehat{L}(B \bullet B), \\
& \int_{M} \frac{\widehat{L}(T M) \operatorname{ch}\left(T_{\mathbf{C}} M\right)}{\cosh ^{2}\left(\frac{c}{2}\right)} \\
& =\int_{M} \widehat{L}(T M) \operatorname{ch}\left(T_{\mathbf{C}} M\right)-\int_{M} \widehat{L}(T M) \operatorname{ch}\left(T_{\mathbf{C}} M\right) \frac{\sinh ^{2}\left(\frac{c}{2}\right)}{\cosh ^{2}\left(\frac{c}{2}\right)} \\
& =\int_{M} \widehat{L}(T M) \operatorname{ch}\left(T_{\mathbf{C}} M\right)-\int_{B \bullet B} \widehat{L}(B \bullet B) \operatorname{ch}\left(T_{\mathbf{C}}(B \bullet B) \oplus N_{\mathbf{C}}\right),
\end{aligned}
$$

and

$$
\int_{M} \widehat{L}(T M) \operatorname{ch}\left(2 \xi_{\mathbf{C}} \oplus \mathbf{C}^{8}\right) \frac{\sinh ^{2}\left(\frac{c}{2}\right)}{\cosh ^{2}\left(\frac{c}{2}\right)}=\int_{B \bullet B} \widehat{L}(B \bullet B) \operatorname{ch}\left(N_{\mathbf{C}} \oplus \mathbf{C}^{8}\right) .
$$

Therefore, by (2.31), (4.1) to (4.3), we have

$$
\int_{M} \widehat{L}(T M) \operatorname{ch}\left(T_{\mathbf{C}} M\right)-\int_{B \bullet B} \widehat{L}(B \bullet B) \operatorname{ch}\left(T_{\mathbf{C}}(B \bullet B) \oplus 2 N_{\mathbf{C}} \oplus \mathbf{C}^{8}\right)
$$

$$
-16\left(\int_{M} \widehat{L}(T M)-\int_{B \bullet B} \widehat{L}(B \bullet B)\right)=2^{14} \int_{M} \sum_{r=0}^{k-1}(k-r) 2^{6(k-r-1)} h_{r}\left(T_{\mathbf{C}} M, \xi_{\mathbf{C}}\right) .
$$

Thus one has

$$
\begin{aligned}
& \frac{1}{128}\left\{\int_{M} \widehat{L}(T M) \operatorname{ch}\left(T_{\mathbf{C}} M\right)-\int_{B \bullet B} \widehat{L}(B \bullet B) \operatorname{ch}\left(T_{\mathbf{C}}(B \bullet B) \oplus 2 N_{\mathbf{C}} \oplus \mathbf{C}^{8}\right)\right\} \\
= & \frac{\int_{M} \widehat{L}(T M)-\int_{B \bullet B} \widehat{L}(B \bullet B)}{8}+2^{7} \int_{M} \sum_{r=0}^{k-1}(k-r) 2^{6(k-r-1)} h_{r}\left(T_{\mathbf{C}} M, \xi_{\mathbf{C}}\right),
\end{aligned}
$$

and

$$
\begin{aligned}
& \frac{1}{2^{14}}\left\{\int_{M} \widehat{L}(T M) \operatorname{ch}\left(T_{\mathbf{C}} M\right)-\int_{B \bullet B} \widehat{L}(B \bullet B) \operatorname{ch}\left(T_{\mathbf{C}}(B \bullet B) \oplus 2 N_{\mathbf{C}} \oplus \mathbf{C}^{8}\right)\right. \\
& \left.-16\left(\int_{M} \widehat{L}(T M)-\int_{B \bullet B} \widehat{L}(B \bullet B)\right)\right\} \\
= & \int_{M} \sum_{r=0}^{k-1}(k-r) 2^{6(k-r-1)} h_{r}\left(T_{\mathbf{C}} M, \xi_{\mathbf{C}}\right) \\
= & \int_{M} \sum_{r=0}^{k-2}(k-r) 2^{6(k-r-1)} h_{r}\left(T_{\mathbf{C}} M, \xi_{\mathbf{C}}\right)+\int_{M} h_{k-1}\left(T_{\mathbf{C}} M, \xi_{\mathbf{C}}\right) \\
= & \int_{M} \sum_{r=0}^{k-2}(k-r) 2^{6(k-r-1)} h_{r}\left(T_{\mathbf{C}} M, \xi_{\mathbf{C}}\right) \\
& +\int_{M} \widehat{A}\left(T M, \nabla^{T M}\right) \operatorname{ch}\left(b_{k-1}\left(T_{\mathbf{C}} M, \xi_{\mathbf{C}}\right)\right) \cosh \left(\frac{c}{2}\right) .
\end{aligned}
$$


From [9, Theorem 3.2] (Theorem 4.2 below), Theorem 2.5, (4.5), (4.6) and the Atiyah-Singer index theorem for $\operatorname{spin}^{c}$ manifolds, we obtain that

Theorem 4.1. If $M$ is an $8 k+4$ dimensional closed $\operatorname{spin}^{c}$ manifold, $\xi$ is a complex line bundle over $M$ such that $c_{1}(\xi) \equiv w_{2}(T M) \in H^{2}\left(M, \mathbf{Z}_{2}\right)$ and $B$ is an oriented $8 k+2$ dimensional submanifold of $M$ such that $[B] \in H_{8 k+2}(M, \mathbf{Z})$ is dual to $c_{1}(\xi) \in H^{2}(M, \mathbf{Z})$, then

$$
\operatorname{Sig}\left(M, T_{\mathbf{C}} M\right)-\operatorname{Sig}\left(B \bullet B, T_{\mathbf{C}}(B \bullet B) \oplus 2 N_{\mathbf{C}} \oplus \mathbf{C}^{8}\right)
$$

is divisible by 128 and

$$
\operatorname{Sig}\left(M, T_{\mathbf{C}} M\right)-\operatorname{Sig}\left(B \bullet B, T_{\mathbf{C}}(B \bullet B) \oplus 2 N_{\mathbf{C}} \oplus \mathbf{C}^{8}\right)-16(\operatorname{Sig}(M)-\operatorname{Sig}(B \bullet B))
$$

is divisible by $2^{14}$. Moreover, one has

$$
\begin{aligned}
& \frac{1}{128}\left\{\operatorname{Sig}\left(M, T_{\mathbf{C}} M\right)-\operatorname{Sig}\left(B \bullet B, T_{\mathbf{C}}(B \bullet B) \oplus 2 N_{\mathbf{C}} \oplus \mathbf{C}^{8}\right)\right\} \\
\equiv & \int_{M} \widehat{A}\left(T M, \nabla^{T M}\right) \operatorname{ch}\left(b_{k}\left(T_{\mathbf{C}} M, \xi_{\mathbf{C}}\right)\right) \cosh \left(\frac{c}{2}\right) \quad \bmod 64,
\end{aligned}
$$

and

$$
\begin{aligned}
& \frac{1}{2^{14}}\left\{\operatorname{Sig}\left(M, T_{\mathbf{C}} M\right)-\operatorname{Sig}\left(B \bullet B, T_{\mathbf{C}}(B \bullet B) \oplus 2 N_{\mathbf{C}} \oplus \mathbf{C}^{8}\right)\right. \\
&-16(\operatorname{Sig}(M)-\operatorname{Sig}(B \bullet B))\} \\
& \equiv \int_{M} \widehat{A}\left(T M, \nabla^{T M}\right) \operatorname{ch}\left(b_{k-1}\left(T_{\mathbf{C}} M, \xi_{\mathbf{C}}\right)\right) \cosh \left(\frac{c}{2}\right) \bmod 128
\end{aligned}
$$

Note that we also have the following results.

Theorem 4.2 (Han-Zhang [9, Theorem 3.2]). The following congruence formula holds:

$$
\begin{aligned}
& \frac{\operatorname{Sig}(M)-\operatorname{Sig}(B \bullet B)}{8} \\
\equiv & \int_{M} \widehat{A}\left(T M, \nabla^{T M}\right) \operatorname{ch}\left(b_{k}\left(T_{\mathbf{C}} M, \xi_{\mathbf{C}}\right)\right) \cosh \left(\frac{c}{2}\right) \bmod 64
\end{aligned}
$$

Theorem 4.3 (Liu-Zhang [18, Theorem 4.2], Han-Zhang 9, formulas 3.5, 3.6 and 3.14$]$ ). The following congruence formulas hold:

$$
\begin{aligned}
& \int_{M} \widehat{A}\left(T M, \nabla^{T M}\right) \operatorname{ch}\left(b_{r}\left(T_{\mathbf{C}} M, \xi_{\mathbf{C}}\right)\right) \cosh \left(\frac{c}{2}\right) \\
\equiv & \int_{M} \widehat{A}\left(T M, \nabla^{T M}\right) \operatorname{ch}\left(b_{r}\left(T_{\mathbf{C}} M+\mathbf{C}^{2}-\xi_{\mathbf{C}}, \mathbf{C}^{2}\right)\right) \cosh \left(\frac{c}{2}\right) \bmod 2,
\end{aligned}
$$

$0 \leq r \leq k$, and

$$
\begin{aligned}
& \frac{\operatorname{Sig}(M)-\operatorname{Sig}(B \bullet B)}{8} \\
\equiv & \int_{M} \widehat{A}\left(T M, \nabla^{T M}\right) \operatorname{ch}\left(b_{k}\left(T_{\mathbf{C}} M+\mathbf{C}^{2}-\xi_{\mathbf{C}}, \mathbf{C}^{2}\right)\right) \cosh \left(\frac{c}{2}\right) \bmod 2 .
\end{aligned}
$$

Let $E$ be a real vector bundle over $M$ and $i: B \hookrightarrow M$ denote the canonical embedding of $B$ in $M$. Then we have the following theorem. 
Theorem 4.4 (Zhang, [20]). The following identity holds:

$$
\int_{M} \widehat{A}\left(T M, \nabla^{T M}\right) \operatorname{ch}(E \otimes \mathbf{C}) \cosh \left(\frac{c}{2}\right) \equiv \operatorname{ind}_{2}\left(i^{*} E\right) \quad \bmod 2,
$$

where $\operatorname{ind}_{2}\left(i^{*} E\right)$ is the mod 2 index in the sense of Atiyah and Singer.

Note that Theorem 4.4 only holds for $8 k+4$ dimensional spin ${ }^{c}$ manifolds.

Combining Theorems 4.1, 4.3 and 4.4, we obtain that

Corollary 4.1. The following congruence formulas hold:

$$
\begin{aligned}
& \frac{1}{128}\left\{\operatorname{Sig}\left(M, T_{\mathbf{C}} M\right)-\operatorname{Sig}\left(B \bullet B, T_{\mathbf{C}}(B \bullet B) \oplus 2 N_{\mathbf{C}} \oplus \mathbf{C}^{8}\right)\right\} \\
\equiv & \operatorname{ind}_{2}\left(b_{k}\left(T B+\mathbf{R}^{2}, \mathbf{R}^{2}\right)\right) \quad \bmod 2,
\end{aligned}
$$

and

$$
\begin{aligned}
\frac{1}{2^{14}}\left\{\operatorname{Sig}\left(M, T_{\mathbf{C}} M\right)-\operatorname{Sig}(B \bullet B\right. & \left.T_{\mathbf{C}}(B \bullet B) \oplus 2 N_{\mathbf{C}} \oplus \mathbf{C}^{8}\right) \\
& -16(\operatorname{Sig}(M)-\operatorname{Sig}(B \bullet B))\} \\
& \equiv \operatorname{ind}_{2}\left(b_{k-1}\left(T B+\mathbf{R}^{2}, \mathbf{R}^{2}\right)\right) \quad \bmod 2 .
\end{aligned}
$$

We can regard (4.13) and (4.14) as twisted Rokhlin congruence formulas for $8 k+4$ dimensional $\operatorname{spin}^{c}$ manifolds.

4.2. $8 k$ dimensional case. By similar computations as what we did in the above subsection, for the $8 k$ dimensional case, from Theorem 2.6 , we have

$$
\begin{aligned}
& \int_{M} \widehat{L}(T M) \operatorname{ch}\left(T_{\mathbf{C}} M\right)-\int_{B \bullet B} \widehat{L}(B \bullet B) \operatorname{ch}\left(T_{\mathbf{C}}(B \bullet B) \oplus 2 N_{\mathbf{C}} \oplus \mathbf{C}^{8}\right) \\
= & \left.2^{11} \int_{M} \sum_{r=0}^{k-1}(k-r) 2^{6(k-r-1)} z_{r}\left(T_{\mathbf{C}} M, \xi_{\mathbf{C}}\right)\right) .
\end{aligned}
$$

Theorem 4.5. Let $M$ be an $8 k$ dimensional closed spin ${ }^{c}$ manifold, $\xi$ is a complex line bundle over $M$ such that $c_{1}(\xi) \equiv w_{2}(T M) \in H^{2}\left(M, \mathbf{Z}_{2}\right)$ and $B$ is an oriented $8 k-2$ dimensional submanifold of $M$ such that $[B] \in H_{8 k-2}(M, \mathbf{Z})$ is dual to $c_{1}(\xi) \in H^{2}(M, \mathbf{Z})$. Let $B \bullet B$ be the self-intersection to $B$ in $M$ and $N$ be the normal bundle of $B \bullet B$ in $M$. Then

$$
\operatorname{Sig}\left(M, T_{\mathbf{C}} M\right)-\operatorname{Sig}\left(B \bullet B, T_{\mathbf{C}}(B \bullet B) \oplus 2 N_{\mathbf{C}} \oplus \mathbf{C}^{8}\right)
$$

is divisible by 2048. Moreover, one has

$$
\begin{aligned}
& \frac{1}{2048}\left\{\operatorname{Sig}\left(M, T_{\mathbf{C}} M\right)-\operatorname{Sig}\left(B \bullet B, T_{\mathbf{C}}(B \bullet B) \oplus 2 N_{\mathbf{C}} \oplus \mathbf{C}^{8}\right)\right\} \\
\equiv & \int_{M} z_{k-1}\left(T_{\mathbf{C}} M, \xi_{\mathbf{C}}\right)=\int_{M} \widehat{A}\left(T M, \nabla^{T M}\right) \operatorname{ch}\left(d_{k-1}\left(T_{\mathbf{C}} M, \xi_{\mathbf{C}}\right)\right) \cosh \left(\frac{c}{2}\right) \bmod 128 .
\end{aligned}
$$

Remark 4.1. It's pretty interesting to note that in the $8 k$ dimensional $\operatorname{spin}^{c}$ case, although we can say nothing about the divisibility of $(\operatorname{Sig}(M)-\operatorname{Sig}(B \bullet B))$, we do have a very high divisibility for the twisted version

$$
\operatorname{Sig}\left(M, T_{\mathbf{C}} M\right)-\operatorname{Sig}\left(B \bullet B, T_{\mathbf{C}}(B \bullet B) \oplus 2 N_{\mathbf{C}} \oplus \mathbf{C}^{8}\right),
$$

which is even much higher than the $8 k+4$ dimensional case. 


\section{Proofs of tWisted CANCELlation FORMUlas}

We use the modular invariance method developed in [16, 8, 9] to prove Theorem 2.1 to 2.6 in this section.

We first recall some necessary knowledge on theta-functions and modular forms. Then in Section 5.1 we prove Theorems 2.1 to 2.4 together and in Section 5.2 we prove Theorems 2.5 and 2.6 together.

Recall that the four Jacobi theta-functions [5] defined by infinite multiplications are

$$
\begin{gathered}
\theta(v, \tau)=2 q^{1 / 8} \sin (\pi v) \prod_{j=1}^{\infty}\left[\left(1-q^{j}\right)\left(1-e^{2 \pi \sqrt{-1} v} q^{j}\right)\left(1-e^{-2 \pi \sqrt{-1} v} q^{j}\right)\right] \\
\theta_{1}(v, \tau)=2 q^{1 / 8} \cos (\pi v) \prod_{j=1}^{\infty}\left[\left(1-q^{j}\right)\left(1+e^{2 \pi \sqrt{-1} v} q^{j}\right)\left(1+e^{-2 \pi \sqrt{-1} v} q^{j}\right)\right], \\
\theta_{2}(v, \tau)=\prod_{j=1}^{\infty}\left[\left(1-q^{j}\right)\left(1-e^{2 \pi \sqrt{-1} v} q^{j-1 / 2}\right)\left(1-e^{-2 \pi \sqrt{-1} v} q^{j-1 / 2}\right)\right], \\
\theta_{3}(v, \tau)=\prod_{j=1}^{\infty}\left[\left(1-q^{j}\right)\left(1+e^{2 \pi \sqrt{-1} v} q^{j-1 / 2}\right)\left(1+e^{-2 \pi \sqrt{-1} v} q^{j-1 / 2}\right)\right],
\end{gathered}
$$

where $q=e^{2 \pi \sqrt{-1} \tau}, \tau \in \mathbf{H}$.

They are all holomorphic functions for $(v, \tau) \in \mathbf{C} \times \mathbf{H}$, where $\mathbf{C}$ is the complex plane and $\mathbf{H}$ is the upper half plane.

Let $\theta^{\prime}(0, \tau)=\left.\frac{\partial}{\partial v} \theta(v, \tau)\right|_{v=0}$. Then the following Jacobi identity relates the four theta-functions gracefully.

Proposition 5.1 (Jacobi identity, 5, Chapter 3]). The following identity holds:

$$
\theta^{\prime}(0, \tau)=\pi \theta_{1}(0, \tau) \theta_{2}(0, \tau) \theta_{3}(0, \tau) .
$$

Let

$$
S L_{2}(\mathbf{Z}):=\left\{\left(\begin{array}{ll}
a & b \\
c & d
\end{array}\right) \mid a, b, c, d \in \mathbf{Z}, a d-b c=1\right\}
$$

as usual be the famous modular group. Let

$$
S=\left(\begin{array}{cc}
0 & -1 \\
1 & 0
\end{array}\right), \quad T=\left(\begin{array}{ll}
1 & 1 \\
0 & 1
\end{array}\right)
$$

be the two generators of $S L_{2}(\mathbf{Z})$. Their actions on $\mathbf{H}$ are given by

$$
S: \tau \rightarrow-\frac{1}{\tau}, \quad T: \tau \rightarrow \tau+1
$$

Let

$$
\begin{aligned}
& \Gamma_{0}(2)=\left\{\left(\begin{array}{ll}
a & b \\
c & d
\end{array}\right) \in S L_{2}(\mathbf{Z}) \mid c \equiv 0 \quad(\bmod 2)\right\}, \\
& \Gamma^{0}(2)=\left\{\left(\begin{array}{ll}
a & b \\
c & d
\end{array}\right) \in S L_{2}(\mathbf{Z}) \mid b \equiv 0(\bmod 2)\right\}
\end{aligned}
$$

be the two modular subgroups of $S L_{2}(\mathbf{Z})$. It is known that the generators of $\Gamma_{0}(2)$ are $T, S T^{2} S T$, while the generators of $\Gamma^{0}(2)$ are $S T S, T^{2} S T S$ (cf. [5]). 
If we let $S$ and $T$ act on the theta-functions, the following transformation formulas hold (cf. [5]):

$$
\theta(v, \tau+1)=e^{\frac{\pi \sqrt{-1}}{4}} \theta(v, \tau), \quad \theta(v,-1 / \tau)=\frac{1}{\sqrt{-1}}\left(\frac{\tau}{\sqrt{-1}}\right)^{1 / 2} e^{\pi \sqrt{-1} \tau v^{2}} \theta(\tau v, \tau)
$$

$$
\theta_{1}(v, \tau+1)=e^{\frac{\pi \sqrt{-1}}{4}} \theta_{1}(v, \tau), \quad \theta_{1}(v,-1 / \tau)=\left(\frac{\tau}{\sqrt{-1}}\right)^{1 / 2} e^{\pi \sqrt{-1} \tau v^{2}} \theta_{2}(\tau v, \tau)
$$

$$
\begin{aligned}
& \theta_{2}(v, \tau+1)=\theta_{3}(v, \tau), \quad \theta_{2}(v,-1 / \tau)=\left(\frac{\tau}{\sqrt{-1}}\right)^{1 / 2} e^{\pi \sqrt{-1} \tau v^{2}} \theta_{1}(\tau v, \tau) \\
& \theta_{3}(v, \tau+1)=\theta_{2}(v, \tau), \quad \theta_{3}(v,-1 / \tau)=\left(\frac{\tau}{\sqrt{-1}}\right)^{1 / 2} e^{\pi \sqrt{-1} \tau v^{2}} \theta_{3}(\tau v, \tau) .
\end{aligned}
$$

Let $\Gamma$ be a subgroup of $S L_{2}(\mathbf{Z})$.

Definition 5.1. A modular form over $\Gamma$ is a holomorphic function $f(\tau)$ on $\mathbf{H} \cup\{\infty\}$ such that for any

$$
g=\left(\begin{array}{ll}
a & b \\
c & d
\end{array}\right) \in \Gamma
$$

the following property holds:

$$
f(g \tau):=f\left(\frac{a \tau+b}{c \tau+d}\right)=\chi(g)(c \tau+d)^{k} f(\tau),
$$

where $\chi: \Gamma \rightarrow \mathbf{C}^{*}$ is a character of $\Gamma$ and $k$ is called the weight of $f$.

If $\Gamma$ is a modular subgroup, let $\mathcal{M}_{\mathbf{R}}(\Gamma)$ denote the ring of modular forms over $\Gamma$ with real Fourier coefficients. Writing simply $\theta_{j}=\theta_{j}(0, \tau), 1 \leq j \leq 3$, we introduce four explicit modular forms (cf. [16]),

$$
\begin{aligned}
\delta_{1}(\tau) & =\frac{1}{8}\left(\theta_{2}^{4}+\theta_{3}^{4}\right), & \varepsilon_{1}(\tau) & =\frac{1}{16} \theta_{2}^{4} \theta_{3}^{4}, \\
\delta_{2}(\tau) & =-\frac{1}{8}\left(\theta_{1}^{4}+\theta_{3}^{4}\right), & \varepsilon_{2}(\tau) & =\frac{1}{16} \theta_{1}^{4} \theta_{3}^{4} .
\end{aligned}
$$

They have the following Fourier expansions in $q^{1 / 2}$ :

$$
\begin{aligned}
& \delta_{1}(\tau)=\frac{1}{4}+6 q+6 q^{2}+\cdots, \quad \varepsilon_{1}(\tau)=\frac{1}{16}-q+7 q^{2}+\cdots, \\
& \delta_{2}(\tau)=-\frac{1}{8}-3 q^{1 / 2}-3 q+\cdots, \quad \varepsilon_{2}(\tau)=q^{1 / 2}+8 q+\cdots .
\end{aligned}
$$

where the "..." terms are the higher degree terms, all of which have integral coefficients. They also satisfy the transformation laws (cf. [14], 16]),

$$
\delta_{2}\left(-\frac{1}{\tau}\right)=\tau^{2} \delta_{1}(\tau), \quad \varepsilon_{2}\left(-\frac{1}{\tau}\right)=\tau^{4} \varepsilon_{1}(\tau) .
$$

Lemma 5.1 (16]). One has that $\delta_{1}(\tau)$ (resp. $\left.\varepsilon_{1}(\tau)\right)$ is a modular form of weight 2 (resp. 4) over $\Gamma_{0}(2)$, while $\delta_{2}(\tau)$ (resp. $\varepsilon_{2}(\tau)$ ) is a modular form of weight 2 (resp. 4) over $\Gamma^{0}(2)$, and moreover $\mathcal{M}_{\mathbf{R}}\left(\Gamma^{0}(2)\right)=\mathbf{R}\left[\delta_{2}(\tau), \varepsilon_{2}(\tau)\right]$. 
5.1. Proof of Theorems 2.1 to 2.4. Without loss of generality, we will adopt the Chern roots formalism as in [16, in the computations of characteristic forms.

Recall that if $\left\{w_{i}\right\}$ are the formal Chern roots of a Hermitian vector bundle $E$ carrying a Hermitian $\nabla^{E}$, then one has the following formula for the Chern character form of the exterior power of $E$ [1]:

$$
\operatorname{ch}\left(\Lambda_{t}(E)\right)=\prod_{i}\left(1+e^{w_{i}} t\right) .
$$

Let's deal with $8 k+4$ dimensional manifolds first.

For $\tau \in \mathbf{H}$ and $q=e^{2 \pi \sqrt{-1} \tau}$, set (cf. [16])

$$
\begin{aligned}
& P_{1}(\tau)=\left\{\widehat{L}\left(T M, \nabla^{T M}\right) \operatorname{ch}\left(\Theta_{1}\left(T_{\mathbf{C}} M\right), \nabla^{\Theta_{1}\left(T_{\mathbf{C}} M\right)}\right)\right\}^{(8 k+4)} \\
& P_{2}(\tau)=\left\{\widehat{A}\left(T M, \nabla^{T M}\right) \operatorname{ch}\left(\Theta_{2}\left(T_{\mathbf{C}} M\right), \nabla^{\Theta_{2}\left(T_{\mathbf{C}} M\right)}\right)\right\}^{(8 k+4)}
\end{aligned}
$$

where $\nabla^{\Theta_{i}\left(T_{\mathbf{C}} M\right)}, i=1,2$, are the Hermitian connections with $q^{j / 2}$-coefficients on $\Theta_{i}\left(T_{\mathbf{C}} M\right)$ induced from those on the $A_{j}\left(T_{\mathbf{C}} M\right)$ 's and $B_{j}\left(T_{\mathbf{C}} M\right)$ 's.

Let $\left\{ \pm 2 \pi \sqrt{-1} x_{j}\right\}$ be the formal Chern roots for $\left(T_{\mathbf{C}} M, \nabla^{T_{\mathbf{C}} M}\right)$. In terms of the theta-functions, we get (cf. [16])

$$
\begin{gathered}
P_{1}(\tau)=2^{4 k+2}\left\{\prod_{j=1}^{4 k+2} x_{j} \frac{\theta^{\prime}(0, \tau)}{\theta\left(x_{j}, \tau\right)} \frac{\theta_{1}\left(x_{j}, \tau\right)}{\theta_{1}(0, \tau)}\right\}^{(8 k+4)}, \\
P_{2}(\tau)=\left\{\prod_{j=1}^{4 k+2} x_{j} \frac{\theta^{\prime}(0, \tau)}{\theta\left(x_{j}, \tau\right)} \frac{\theta_{2}\left(x_{j}, \tau\right)}{\theta_{2}(0, \tau)}\right\}^{(8 k+4)} .
\end{gathered}
$$

Applying the transformation laws (5.6) to (5.9) for theta-functions, we see that $P_{1}(\tau)$ is a modular form of weight $4 k+2$ over $\Gamma_{0}(2)$, while $P_{2}(\tau)$ is a modular form of weight $4 k+2$ over $\Gamma^{0}(2)$. Moreover, the following identity holds:

$$
P_{1}(-1 / \tau)=(2 \tau)^{4 k+2} P_{2}(\tau) .
$$

Observe that at any point $x \in M$, up to the volume form determined by the metric on $T_{x} M$, both $P_{i}(\tau), i=1,2$, can be viewed as a power series of $q^{1 / 2}$ with real Fourier coefficients. Thus, one can apply Lemma 5.1 to $P_{2}(\tau)$ to get, at $x$, that

$$
P_{2}(\tau)=h_{0}\left(T_{\mathbf{C}} M\right)\left(8 \delta_{2}\right)^{2 k+1}+h_{1}\left(T_{\mathbf{C}} M\right)\left(8 \delta_{2}\right)^{2 k-1} \varepsilon_{2}+\cdots+h_{k}\left(T_{\mathbf{C}} M\right)\left(8 \delta_{2}\right) \varepsilon_{2}^{k},
$$

where each $h_{r}\left(T_{\mathbf{C}} M\right), 0 \leq r \leq k$, is a real multiple of the volume form at $x$.

We can show that each $h_{r}\left(T_{\mathbf{C}} M\right), 0 \leq r \leq k$, can be expressed through a canonical integral linear combination of $\left\{\widehat{A}\left(T M, \nabla^{T M}\right) \operatorname{ch}\left(B_{j}\left(T_{\mathbf{C}} M\right), \nabla^{B_{j}\left(T_{\mathbf{C}} M\right)}\right)\right\}^{(8 k+4)}$, $0 \leq j \leq r$, with coefficients not depending on $x \in M$. As in 16, one can use the induction method to prove this fact easily by comparing the coefficients of $q^{j / 2}, j \geq 0$, between the two sides of (5.17). For the consideration of the length of this paper, we do not give details here but only write down the explicit expressions for $h_{0}\left(T_{\mathbf{C}} M\right)$ 
and $h_{1}\left(T_{\mathbf{C}} M\right)$ as follows:

$$
h_{1}\left(T_{\mathbf{C}} M\right)=\left\{\widehat{A}\left(T M, \nabla^{T M}\right)\left[24(2 k+1)-\operatorname{ch}\left(B_{1}\left(T_{\mathbf{C}} M\right), \nabla^{B_{1}\left(T_{\mathbf{C}} M\right)}\right)\right]\right\}^{(8 k+4)} .
$$

By (5.16) and (5.17), we have

$$
\begin{aligned}
& P_{1}(\tau)=2^{4 k+2} \frac{1}{\tau^{4 k+2}} P_{2}(-1 / \tau) \\
& =2^{4 k+2} \frac{1}{\tau^{4 k+2}}\left[h_{0}\left(T_{\mathbf{C}} M\right)\left(8 \delta_{2}(-1 / \tau)\right)^{2 k+1}\right. \\
& +h_{1}\left(T_{\mathbf{C}} M\right)\left(8 \delta_{2}(-1 / \tau)\right)^{2 k-1} \varepsilon_{2}(-1 / \tau)+\cdots \\
& \left.+h_{k}\left(T_{\mathbf{C}} M\right)\left(8 \delta_{2}(-1 / \tau)\right)\left(\varepsilon_{2}(-1 / \tau)\right)^{k}\right] \\
& =2^{4 k+2}\left[h_{0}\left(T_{\mathbf{C}} M\right)\left(8 \delta_{1}\right)^{2 k+1}+h_{1}\left(T_{\mathbf{C}} M\right)\left(8 \delta_{1}\right)^{2 k-1} \varepsilon_{1}+\cdots\right. \\
& \left.+h_{k}\left(T_{\mathbf{C}} M\right)\left(8 \delta_{1}\right) \varepsilon_{1}^{k}\right] .
\end{aligned}
$$

Expanding $\Theta_{1}\left(T_{\mathbf{C}} M\right)$ explicitly, by (2.8) we have

$$
\begin{aligned}
\Theta_{1}\left(T_{\mathbf{C}} M\right)= & \bigotimes_{n=1}^{\infty} S_{q^{n}}\left(T_{\mathbf{C}} M\right) \Lambda_{-q^{n}}\left(\mathbf{C}^{8 k+4}\right) \otimes \bigotimes_{m=1}^{\infty} \Lambda_{q^{m}}\left(T_{\mathbf{C}} M\right) S_{-q^{m}}\left(\mathbf{C}^{8 k+4}\right) \\
= & \left(1+\left(T_{\mathbf{C}} M\right) q+\left(S^{2} T_{\mathbf{C}} M\right) q^{2}+\cdots\right)\left(1+\left(T_{\mathbf{C}} M\right) q^{2}+\cdots\right) \\
& \times\left(1-\mathbf{C}^{8 k+4} q+\left(\Lambda^{2} \mathbf{C}^{8 k+4}\right) q^{2}+\cdots\right)\left(1-\mathbf{C}^{8 k+4} q^{2}+\cdots\right) \\
& \times\left(1+\left(T_{\mathbf{C}} M\right) q+\left(\Lambda^{2} T_{\mathbf{C}} M\right) q^{2}+\cdots\right)\left(1+\left(T_{\mathbf{C}} M\right) q^{2}+\cdots\right) \\
& \times\left(1-\mathbf{C}^{8 k+4} q+\left(S^{2} \mathbf{C}^{8 k+4}\right) q^{2}+\cdots\right)\left(1-\mathbf{C}^{8 k+4} q^{2}+\cdots\right) \\
= & \left(1+2\left(T_{\mathbf{C}} M\right) q+\left(T_{\mathbf{C}} M \otimes T_{\mathbf{C}} M+S^{2} T_{\mathbf{C}} M+\Lambda^{2} T_{\mathbf{C}} M\right) q^{2}+\cdots\right) \\
& \times\left(1+2\left(T_{\mathbf{C}} M\right) q^{2}+\cdots\right) \\
& \times\left(1-2 \mathbf{C}^{8 k+4} q+\left(\mathbf{C}^{8 k+4} \otimes \mathbf{C}^{8 k+4}+S^{2} \mathbf{C}^{8 k+4}+\Lambda^{2} \mathbf{C}^{8 k+4}\right) q^{2}+\cdots\right) \\
& \times\left(1-2 \mathbf{C}^{8 k+4} q^{2}+\cdots\right) \\
= & \left(1+2\left(T_{\mathbf{C}} M\right) q+2\left(T_{\mathbf{C}} M+T_{\mathbf{C}} M \otimes T_{\mathbf{C}} M\right) q^{2}+\cdots\right) \\
& \times\left(1-2 \mathbf{C}^{8 k+4} q+2\left(\mathbf{C}^{8 k+4} \otimes \mathbf{C}^{8 k+4}-\mathbf{C}^{8 k+4}\right) q^{2}+\cdots\right) \\
= & 1+2\left(T_{\mathbf{C}} M-\mathbf{C}^{8 k+4}\right) q \\
& +2\left[-(16 k+7) T_{\mathbf{C}} M+T_{\mathbf{C}} M \otimes T_{\mathbf{C}} M+(8 k+4)(8 k+3)\right] q^{2}+\cdots,
\end{aligned}
$$

where the "..." are the terms involving $q^{j}$ 's with $j \geq 3$. 
Note that

$$
\begin{aligned}
& \left(8 \delta_{1}\right)^{2 k+1-2 r} \varepsilon_{1}{ }^{r} \\
= & \left(2+48 q+48 q^{2} \cdots\right)^{2 k+1-2 r}\left(\frac{1}{16}-q+7 q^{2} \cdots\right)^{r} \\
= & 2^{2 k+1-6 r}\left[1+24(2 k+1-2 r) q+24(2 k+1-2 r)(24 k-24 r+1) q^{2} \cdots\right] \\
& \times\left[1-16 r q+16\left(8 r^{2}-r\right) q^{2} \cdots\right] \\
= & 2^{2 k+1-6 r}[1+(48 k+24-64 r) q+ \\
& \left.\times\left(1152 k^{2}-3072 k r+2048 r^{2}+624 k-1024 r+24\right) q^{2}+\cdots\right] .
\end{aligned}
$$

Therefore, by (5.12), (5.21) and (5.22), setting $q=0$ in (5.20), we get the result of Liu ([16])

$$
\left\{\widehat{L}\left(T M, \nabla^{T M}\right)\right\}^{(8 k+4)}=8 \sum_{r=0}^{k} 2^{6 k-6 r} h_{r}\left(T_{\mathbf{C}} M\right)
$$

On the other hand, by (5.12), (5.21) and (5.22), comparing the coefficients of $q$ in (5.20), we have

$$
\begin{aligned}
& \left\{\widehat{L}\left(T M, \nabla^{T M}\right) \operatorname{ch}\left(2\left(T_{\mathbf{C}} M\right)-2(8 k+4)\right)\right\}^{(8 k+4)} \\
= & 8 \sum_{r=0}^{k} h_{r}\left(T_{\mathbf{C}} M\right) 2^{6 k-6 r}(48 k+24-64 r) .
\end{aligned}
$$

Thus

$$
\begin{aligned}
& \left\{\widehat{L}\left(T M, \nabla^{T M}\right) \operatorname{ch}\left(\left(T_{\mathbf{C}} M\right)-(8 k+4)\right)\right\}^{(8 k+4)} \\
= & 4 \sum_{r=0}^{k} h_{r}\left(T_{\mathbf{C}} M\right) 2^{6 k-6 r}(48 k+24-64 r) \\
= & 12\left(8 \sum_{r=0}^{k} 2^{6 k-6 r} h_{r}\left(T_{\mathbf{C}} M\right)\right)-8 k\left(8 \sum_{r=0}^{k} 2^{6 k-6 r} h_{r}\left(T_{\mathbf{C}} M\right)\right) \\
& +\sum_{r=0}^{k} h_{r}\left(T_{\mathbf{C}} M\right) 2^{6 k-6 r}(256 k-256 r) \\
= & 12\left(8 \sum_{r=0}^{k} 2^{6 k-6 r} h_{r}\left(T_{\mathbf{C}} M\right)\right)-8 k\left(8 \sum_{r=0}^{k} 2^{6 k-6 r} h_{r}\left(T_{\mathbf{C}} M\right)\right) \\
& +256 \cdot 2^{6} \sum_{r=0}^{k-1}(k-r) h_{r}\left(T_{\mathbf{C}} M\right) 2^{6 k-6 r-6} .
\end{aligned}
$$


Combining (5.23) and (5.25), one has

$$
\begin{aligned}
& \left\{\widehat{L}\left(T M, \nabla^{T_{\mathbf{C}} M}\right) \operatorname{ch}\left(T_{\mathbf{C}} M\right)-16 \widehat{L}\left(T M, \nabla^{T M}\right)\right\}^{(8 k+4)} \\
= & 2^{8}\left[\sum_{r=0}^{k}(k-r) 2^{6(k-r)} h_{r}\left(T_{\mathbf{C}} M\right)\right] \\
= & 2^{14}\left[\sum_{r=0}^{k-1}(k-r) 2^{6(k-r-1)} h_{r}\left(T_{\mathbf{C}} M\right)\right]
\end{aligned}
$$

which is just (2.13).

Furthermore, by (5.12), (5.21) and (5.22), comparing the coefficients of $q^{2}$ in (5.20), we have

$$
\begin{aligned}
& \left\{\widehat{L}\left(T M, \nabla^{T M}\right) \operatorname{ch}\left(2\left[-(16 k+7) T_{\mathbf{C}} M+T_{\mathbf{C}} M \otimes T_{\mathbf{C}} M+\mathbf{C}^{(8 k+4)(8 k+3)}\right]\right)\right\}^{(8 k+4)} \\
= & 8 \sum_{r=0}^{k} h_{r}\left(T_{\mathbf{C}} M\right) 2^{6 k-6 r}\left(1152 k^{2}-3072 k r+2048 r^{2}+624 k-1024 r+24\right) .
\end{aligned}
$$

Thus, combining (5.23) and (5.26), we have

$$
\begin{aligned}
& \left\{\widehat{L}\left(T M, \nabla^{T M}\right) \operatorname{ch}\left(\left[-(16 k+7) T_{\mathbf{C}} M+T_{\mathbf{C}} M \otimes T_{\mathbf{C}} M+\mathbf{C}^{(8 k+4)(8 k+3)}\right]\right)\right\}^{(8 k+4)} \\
= & 32 \sum_{r=0}^{k} h_{r}\left(T_{\mathbf{C}} M\right) 2^{6 k-6 r}\left(144 k^{2}-384 k r+256 r^{2}+78 k-128 r+3\right) \\
= & -16 k \cdot 2^{8}\left[\sum_{r=0}^{k}(k-r) 2^{6(k-r)} h_{r}\left(T_{\mathbf{C}} M\right)\right]+48 \cdot 2^{8}\left[\sum_{r=0}^{k}(k-r) 2^{6(k-r)} h_{r}\left(T_{\mathbf{C}} M\right)\right] \\
& +\left(64 k^{2}-200 k+12\right) \cdot 8 \sum_{r=0}^{k} 2^{6 k-6 r} h_{r}\left(T_{\mathbf{C}} M\right) \\
& +2^{13} \sum_{r=0}^{k}(k-r)(k-r-1) 2^{6(k-r)} h_{r}\left(T_{\mathbf{C}} M\right) \\
= & -16 k\left\{\widehat{L}\left(T M, \nabla^{T_{\mathbf{C}} M}\right) \operatorname{ch}\left(T_{\mathbf{C}} M\right)-16 \widehat{L}\left(T M, \nabla^{T M}\right)\right\}^{(8 k+4)} \\
& +48\left\{\widehat{L}\left(T M, \nabla^{T_{\mathbf{C}} M}\right) \operatorname{ch}\left(T_{\mathbf{C}} M\right)-16 \widehat{L}\left(T M, \nabla^{T M}\right)\right\}^{(8 k+4)} \\
& +\left(64 k^{2}-200 k+12\right)\left\{\widehat{L}\left(T M, \nabla^{T_{\mathbf{C}} M}\right)\right\}^{(8 k+4)} \\
& +2^{13} \sum_{r=0}^{k}(k-r)(k-r-1) 2^{6(k-r)} h_{r}\left(T_{\mathbf{C}} M\right) .
\end{aligned}
$$


Therefore by the above computations, we have

$$
\begin{aligned}
& \left\{\widehat{L}\left(T M, \nabla^{T M}\right) \operatorname{ch}\left(T_{\mathbf{C}} M \otimes T_{\mathbf{C}} M\right)-55 \widehat{L}\left(T M, \nabla^{T M}\right) \operatorname{ch}\left(T_{\mathbf{C}} M\right)\right. \\
& \left.+768 \widehat{L}\left(T M, \nabla^{T M}\right)\right\}^{(8 k+4)} \\
& =2^{13} \sum_{r=0}^{k}(k-r)(k-r-1) 2^{6(k-r)} h_{r}\left(T_{\mathbf{C}} M\right) \\
& =2^{25} \sum_{r=0}^{k-2}(k-r)(k-r-1) 2^{6(k-r-2)} h_{r}\left(T_{\mathbf{C}} M\right),
\end{aligned}
$$

which is just (2.17).

To prove Theorem 2.2 for the $8 k$ dimensional case, similarly we set (cf. [16])

$$
\begin{aligned}
& P_{1}(\tau)=\left\{\widehat{L}\left(T M, \nabla^{T M}\right) \operatorname{ch}\left(\Theta_{1}\left(T_{\mathbf{C}} M\right), \nabla^{\Theta_{1}\left(T_{\mathbf{C}} M\right)}\right)\right\}^{(8 k)}, \\
& P_{2}(\tau)=\left\{\widehat{A}\left(T M, \nabla^{T M}\right) \operatorname{ch}\left(\Theta_{2}\left(T_{\mathbf{C}} M\right), \nabla^{\Theta_{2}\left(T_{\mathbf{C}} M\right)}\right)\right\}^{(8 k)} .
\end{aligned}
$$

Then one similarly finds that $P_{1}(\tau)$ is a modular form of weight $4 k$ over $\Gamma_{0}(2)$, while $P_{2}(\tau)$ is a modular form of weight $4 k$ over $\Gamma^{0}(2)$ and

$$
P_{1}(-1 / \tau)=(2 \tau)^{4 k} P_{2}(\tau)
$$

This time, applying Lemma 5.1, we have

$$
P_{2}(\tau)=z_{0}\left(T_{\mathbf{C}} M\right)\left(8 \delta_{2}\right)^{2 k}+z_{1}\left(T_{\mathbf{C}} M\right)\left(8 \delta_{2}\right)^{2 k-2} \varepsilon_{2}+\cdots+z_{k}\left(T_{\mathbf{C}} M\right) \varepsilon_{2}^{k} .
$$

Thus

$$
P_{1}(\tau)=2^{4 k}\left[z_{0}\left(T_{\mathbf{C}} M\right)\left(8 \delta_{1}\right)^{2 k}+z_{1}\left(T_{\mathbf{C}} M\right)\left(8 \delta_{1}\right)^{2 k-2} \varepsilon_{1}+\cdots+z_{k}\left(T_{\mathbf{C}} M\right) \varepsilon_{1}^{k}\right] .
$$

Now we have

$$
\begin{gathered}
z_{0}\left(T_{\mathbf{C}} M\right)=\left\{\widehat{A}\left(T M, \nabla^{T M}\right)\right\}^{(8 k)} \\
z_{1}\left(T_{\mathbf{C}} M\right)=-\left\{\widehat{A}\left(T M, \nabla^{T M}\right)\left[48 k-\operatorname{ch}\left(B_{1}\left(T_{\mathbf{C}} M\right), \nabla^{B_{1}\left(T_{\mathbf{C}} M\right)}\right)\right]\right\}^{(8 k)}
\end{gathered}
$$


Expanding $\Theta_{1}\left(T_{\mathbf{C}} M\right)$ explicitly, we have

$$
\begin{aligned}
\Theta_{1}\left(T_{\mathbf{C}} M\right)= & \bigotimes_{n=1}^{\infty} S_{q^{n}}\left(T_{\mathbf{C}} M\right) \Lambda_{-q^{n}}\left(\mathbf{C}^{8 k}\right) \otimes \bigotimes_{m=1}^{\infty} \Lambda_{q^{m}}\left(T_{\mathbf{C}} M\right) S_{-q^{m}}\left(\mathbf{C}^{8 k}\right) \\
= & \left(1+\left(T_{\mathbf{C}} M\right) q+\left(S^{2} T_{\mathbf{C}} M\right) q^{2}+\cdots\right)\left(1+\left(T_{\mathbf{C}} M\right) q^{2}+\cdots\right) \\
& \times\left(1-\mathbf{C}^{8 k} q+\left(\Lambda^{2} \mathbf{C}^{8 k}\right) q^{2}+\cdots\right)\left(1-\mathbf{C}^{8 k} q^{2}+\cdots\right) \\
& \times\left(1+\left(T_{\mathbf{C}} M\right) q+\left(\Lambda^{2} T_{\mathbf{C}} M\right) q^{2}+\cdots\right)\left(1+\left(T_{\mathbf{C}} M\right) q^{2}+\cdots\right) \\
& \times\left(1-\mathbf{C}^{8 k} q+\left(S^{2} \mathbf{C}^{8 k}\right) q^{2}+\cdots\right)\left(1-\mathbf{C}^{8 k} q^{2}+\cdots\right) \\
= & \left(1+2\left(T_{\mathbf{C}} M\right) q+\left(T_{\mathbf{C}} M \otimes T_{\mathbf{C}} M+S^{2} T_{\mathbf{C}} M+\Lambda^{2} T_{\mathbf{C}} M\right) q^{2}+\cdots\right) \\
& \times\left(1+2\left(T_{\mathbf{C}} M\right) q^{2}+\cdots\right) \\
& \times\left(1-2 \mathbf{C}^{8 k} q+\left(\mathbf{C}^{8 k} \otimes \mathbf{C}^{8 k}+S^{2} \mathbf{C}^{8 k}+\Lambda^{2} \mathbf{C}^{8 k}\right) q^{2}+\cdots\right) \\
& \times\left(1-2 \mathbf{C}^{8 k} q^{2}+\cdots\right) \\
= & \left(1+2\left(T_{\mathbf{C}} M\right) q+2\left(T_{\mathbf{C}} M+T_{\mathbf{C}} M \otimes T_{\mathbf{C}} M\right) q^{2}+\cdots\right) \\
& \times\left(1-2 \mathbf{C}^{8 k} q+2\left(\mathbf{C}^{8 k} \otimes \mathbf{C}^{8 k}-\mathbf{C}^{8 k}\right) q^{2}+\cdots\right) \\
= & +2\left(T_{\mathbf{C}} M-\mathbf{C}^{8 k}\right) q \\
& +2\left[-(16 k-1) T_{\mathbf{C}} M+T_{\mathbf{C}} M \otimes T_{\mathbf{C}} M+8 k(8 k-1)\right] q^{2}+\cdots
\end{aligned}
$$

where the "..." are the terms involving $q^{j}$ 's with $j \geq 3$. Note that

$$
\begin{aligned}
& \left(8 \delta_{1}\right)^{2 k-2 r} \varepsilon_{1}{ }^{r} \\
= & \left(2+48 q+48 q^{2} \cdots\right)^{2 k-2 r}\left(\frac{1}{16}-q+7 q^{2} \cdots\right)^{r} \\
= & 2^{2 k-6 r}\left[1+24(2 k-2 r) q+24(k-r)(48 k-48 r-22) q^{2} \cdots\right] \\
& \times\left[1-16 r q+16\left(8 r^{2}-r\right) q^{2} \cdots\right] \\
= & 2^{2 k-6 r}[1+(48 k-64 r) q+ \\
& \left.\times\left(1152 k^{2}-3072 k r+2048 r^{2}-528 k+512 r\right) q^{2}+\cdots\right] .
\end{aligned}
$$

Therefore by (5.27), (5.34) and (5.35), comparing the constant terms of both sides of (5.31), we get the result of Liu ([16])

$$
\left\{\widehat{L}\left(T M, \nabla^{T M}\right)\right\}^{(8 k)}=\sum_{r=0}^{k} 2^{6 k-6 r} z_{r}\left(T_{\mathbf{C}} M\right) .
$$


By (5.27), (5.34) and (5.35), comparing the coefficients of $q$ on both sides of (5.31), we have

$$
\begin{aligned}
& \left\{\widehat{L}\left(T M, \nabla^{T M}\right) \operatorname{ch}\left(2\left(T_{\mathbf{C}} M, \nabla^{T_{\mathbf{C}} M}\right)-2(8 k)\right)\right\}^{(8 k)} \\
= & \sum_{r=0}^{k} 2^{6 k-6 r}(48 k-64 r) z_{r}\left(T_{\mathbf{C}} M\right) .
\end{aligned}
$$

Thus

$$
\begin{aligned}
& \left\{\widehat{L}\left(T M, \nabla^{T M}\right) \operatorname{ch}\left(\left(T_{\mathbf{C}} M, \nabla^{T_{\mathbf{C}} M}\right)-8 k\right)\right\}^{(8 k)} \\
= & \sum_{r=0}^{k} 2^{6 k-6 r}(24 k-32 r) z_{r}\left(T_{\mathbf{C}} M\right) \\
= & -8 k\left(\sum_{r=0}^{k} 2^{6 k-6 r} z_{r}\left(T_{\mathbf{C}} M\right)\right)+\sum_{r=0}^{k} 2^{6 k-6 r}(32 k-32 r) z_{r}\left(T_{\mathbf{C}} M\right) \\
= & -8 k\left(\sum_{r=0}^{k} 2^{6 k-6 r} z_{r}\left(T_{\mathbf{C}} M\right)\right)+32 \cdot 2^{6} \sum_{r=0}^{k-1}(k-r) 2^{6 k-6 r-6} z_{r}\left(T_{\mathbf{C}} M\right) .
\end{aligned}
$$

Combining (5.36) and (5.38), we get

$$
\begin{aligned}
& \left\{\widehat{L}\left(T M, \nabla^{T M}\right) \operatorname{ch}\left(T_{\mathbf{C}} M, \nabla^{T_{\mathbf{C}} M}\right)\right\}^{(8 k)} \\
= & 2^{5}\left[\sum_{r=0}^{k}(k-r) 2^{6(k-r)} z_{r}\left(T_{\mathbf{C}} M\right)\right] \\
= & 2^{11}\left[\sum_{r=0}^{k-1}(k-r) 2^{6(k-r-1)} z_{r}\left(T_{\mathbf{C}} M\right)\right]
\end{aligned}
$$

which is just (2.23).

By (5.27), (5.34) and (5.35), comparing the coefficients of $q^{2}$ on both sides of (5.31), we have

$$
\begin{aligned}
& \left\{\widehat{L}\left(T M, \nabla^{T M}\right) \operatorname{ch}\left(2\left[-(16 k-1) T_{\mathbf{C}} M+T_{\mathbf{C}} M \otimes T_{\mathbf{C}} M+\mathbf{C}^{8 k(8 k-1)}\right]\right)\right\}^{(8 k)} \\
= & \sum_{r=0}^{k} z_{r}\left(T_{\mathbf{C}} M\right) 2^{6 k-6 r}\left(1152 k^{2}-3072 k r+2048 r^{2}-528 k+512 r\right) .
\end{aligned}
$$


Thus, combining (5.36) and (5.39), we have

$$
\begin{aligned}
& \left\{\widehat{L}\left(T M, \nabla^{T M}\right) \operatorname{ch}\left(\left[-(16 k-1) T_{\mathbf{C}} M+T_{\mathbf{C}} M \otimes T_{\mathbf{C}} M+\mathbf{C}^{8 k(8 k-1)}\right]\right)\right\}^{(8 k)} \\
= & \sum_{r=0}^{k} z_{r}\left(T_{\mathbf{C}} M\right) 2^{6 k-6 r}\left(576 k^{2}-1536 k r+1024 r^{2}-264 k+256 r\right) \\
= & -16 k \cdot 2^{5}\left[\sum_{r=0}^{k}(k-r) 2^{6(k-r)} z_{r}\left(T_{\mathbf{C}} M\right)\right]+24 \cdot 2^{5}\left[\sum_{r=0}^{k}(k-r) 2^{6(k-r)} z_{r}\left(T_{\mathbf{C}} M\right)\right] \\
& +\left(64 k^{2}-8 k\right) \sum_{r=0}^{k} 2^{6 k-6 r} z_{r}\left(T_{\mathbf{C}} M\right) \\
& +2^{10} \sum_{r=0}^{k}(k-r)(k-r-1) 2^{6(k-r)} z_{r}\left(T_{\mathbf{C}} M\right) \\
= & 16 k\left\{\widehat{L}\left(T M, \nabla^{T_{\mathbf{C}} M}\right) \operatorname{ch}\left(T_{\mathbf{C}} M\right)\right\}^{(8 k)} \\
& +24\left\{\widehat{L}\left(T M, \nabla^{T_{\mathbf{C}} M}\right) \operatorname{ch}\left(T_{\mathbf{C}} M\right)\right\}^{(8 k)} \\
& +\left(64 k^{2}-8 k\right)\left\{\widehat{L}\left(T M, \nabla^{T_{\mathbf{C}} M}\right)\right\}^{(8 k+4)} \\
& +2^{10} \sum_{r=0}^{k}(k-r)(k-r-1) 2^{6(k-r)} z_{r}\left(T_{\mathbf{C}} M\right) .
\end{aligned}
$$

Therefore by the above computations, we have

$$
\begin{aligned}
& \left\{\widehat{L}\left(T M, \nabla^{T M}\right) \operatorname{ch}\left(T_{\mathbf{C}} M \otimes T_{\mathbf{C}} M\right)-23 \widehat{L}\left(T M, \nabla^{T M}\right) \operatorname{ch}\left(T_{\mathbf{C}} M\right)\right\}^{(8 k)} \\
= & 2^{10} \sum_{r=0}^{k}(k-r)(k-r-1) 2^{6(k-r)} z_{r}\left(T_{\mathbf{C}} M\right) \\
= & 2^{22} \sum_{r=0}^{k-2}(k-r)(k-r-1) 2^{6(k-r-2)} z_{r}\left(T_{\mathbf{C}} M\right),
\end{aligned}
$$

which is just (2.26).

5.2. Proof of Theorems 2.5 and 2.6. The proof for the cases with the extra complex line bundle $\xi$ involved is similar to the above proof.

For $\tau \in \mathbf{H}$ and $q=e^{2 \pi \sqrt{-1} \tau}$, set (cf. [8, 9])

$$
P_{1}\left(\xi_{\mathbf{C}}, \tau\right)=\left\{\frac{\widehat{L}\left(T M, \nabla^{T M}\right)}{\cosh ^{2}\left(\frac{c}{2}\right)} \operatorname{ch}\left(\Theta_{1}\left(T_{\mathbf{C}} M, \xi_{\mathbf{C}}\right), \nabla^{\Theta_{1}\left(T_{\mathbf{C}} M, \xi_{\mathbf{C}}\right)}\right)\right\}^{(8 k+4)},
$$

$$
P_{2}\left(\xi_{\mathbf{C}}, \tau\right)=\left\{\widehat{A}\left(T M, \nabla^{T M}\right) \operatorname{ch}\left(\Theta_{2}\left(T_{\mathbf{C}} M, \xi_{\mathbf{C}}\right), \nabla^{\Theta_{2}\left(T_{\mathbf{C}} M, \xi_{\mathbf{C}}\right)}\right) \cosh \left(\frac{c}{2}\right)\right\}^{(8 k+4)},
$$

where $\nabla^{\Theta_{i}\left(T_{\mathbf{C}} M, \xi_{\mathbf{C}}\right)}, i=1,2$, are the Hermitian connections with $q^{j / 2}$-coefficients on $\Theta_{i}\left(T_{\mathbf{C}} M, \xi_{\mathbf{C}}\right)$ induced from those on the $A_{j}\left(T_{\mathbf{C}} M, \xi_{\mathbf{C}}\right)$ 's and $B_{j}\left(T_{\mathbf{C}} M, \xi_{\mathbf{C}}\right)$ 's. 
Let $\left\{ \pm 2 \pi \sqrt{-1} x_{j}\right\}$ be the formal Chern roots for $\left(T_{\mathbf{C}} M, \nabla^{T_{\mathbf{C}} M}\right), c=2 \pi \sqrt{-1} u$. In terms of the theta-functions, we get (cf. [9])

$$
\begin{gathered}
P_{1}\left(\xi_{\mathbf{C}}, \tau\right)=2^{4 k+2}\left\{\left(\prod_{j=1}^{4 k+2} x_{j} \frac{\theta^{\prime}(0, \tau)}{\theta\left(x_{j}, \tau\right)} \frac{\theta_{1}\left(x_{j}, \tau\right)}{\theta_{1}(0, \tau)}\right) \frac{\theta_{1}^{2}(0, \tau)}{\theta_{1}^{2}(u, \tau)} \frac{\theta_{3}(u, \tau)}{\theta_{3}(0, \tau)} \frac{\theta_{2}(u, \tau)}{\theta_{2}(0, \tau)}\right\}^{(8 k+4)}, \\
P_{2}\left(\xi_{\mathbf{C}}, \tau\right)=\left\{\left(\prod_{j=1}^{4 k+2} x_{j} \frac{\theta^{\prime}(0, \tau)}{\theta\left(x_{j}, \tau\right)} \frac{\theta_{2}\left(x_{j}, \tau\right)}{\theta_{2}(0, \tau)}\right) \frac{\theta_{2}^{2}(0, \tau)}{\theta_{2}^{2}(u, \tau)} \frac{\theta_{3}(u, \tau)}{\theta_{3}(0, \tau)} \frac{\theta_{1}(u, \tau)}{\theta_{1}(0, \tau)}\right\}^{(8 k+4)}
\end{gathered}
$$

Applying the transformation laws (5.6) to (5.9) for theta-functions, we still see that ([9]) $P_{1}\left(\xi_{\mathbf{C}}, \tau\right)$ is a modular form of weight $4 k+2$ over $\Gamma_{0}(2)$, while $P_{2}\left(\xi_{\mathbf{C}}, \tau\right)$ is a modular form of weight $4 k+2$ over $\Gamma^{0}(2)$. Moreover, the following identity holds:

$$
P_{1}\left(\xi_{\mathbf{C}},-1 / \tau\right)=(2 \tau)^{4 k+2} P_{2}\left(\xi_{\mathbf{C}}, \tau\right) .
$$

Then similar to (5.17), we have

$$
\begin{aligned}
P_{2}\left(\xi_{\mathbf{C}}, \tau\right)=h_{0}\left(T_{\mathbf{C}} M, \xi_{\mathbf{C}}\right)\left(8 \delta_{2}\right)^{2 k+1}+h_{1}\left(T_{\mathbf{C}} M, \xi_{\mathbf{C}}\right)\left(8 \delta_{2}\right)^{2 k-1} \varepsilon_{2}+\cdots & +h_{k}\left(T_{\mathbf{C}} M, \xi_{\mathbf{C}}\right)\left(8 \delta_{2}\right) \varepsilon_{2}^{k}
\end{aligned}
$$

where each $h_{r}\left(T_{\mathbf{C}} M, \xi_{\mathbf{C}}\right), 0 \leq r \leq k$, can be expressed through a canonical integral linear combination of

$$
\left\{\widehat{A}\left(T M, \nabla^{T M}\right) \operatorname{ch}\left(B_{j}\left(T_{\mathbf{C}} M, \xi_{\mathbf{C}}\right), \nabla^{B_{j}\left(T_{\mathbf{C}} M, \xi_{\mathbf{C}}\right)}\right) \cosh \left(\frac{c}{2}\right)\right\}^{(8 k+4)}, \quad 0 \leq j \leq r .
$$

Explicitly, one has ([9])

$h_{1}\left(T_{\mathbf{C}} M, \xi_{\mathbf{C}}\right)=\left\{\widehat{A}\left(T M, \nabla^{T M}\right)\left[24(2 k+1)-\operatorname{ch}\left(B_{1}\left(T_{\mathbf{C}} M, \xi_{\mathbf{C}}\right)\right)\right] \cosh \left(\frac{c}{2}\right)\right\}^{(8 k+4)}$.

By (5.44) and (5.45), we have

$$
\begin{aligned}
P_{1}\left(\xi_{\mathbf{C}}, \tau\right)= & 2^{4 k+2}\left[h_{0}\left(T_{\mathbf{C}} M, \xi_{\mathbf{C}}\right)\left(8 \delta_{1}\right)^{2 k+1}+h_{1}\left(T_{\mathbf{C}} M, \xi_{\mathbf{C}}\right)\left(8 \delta_{1}\right)^{2 k-1} \varepsilon_{1}\right. \\
& \left.+\cdots+h_{k}\left(T_{\mathbf{C}} M, \xi_{\mathbf{C}}\right)\left(8 \delta_{1}\right) \varepsilon_{1}^{k}\right] .
\end{aligned}
$$


Let's explicitly expand $\Theta_{1}\left(T_{\mathbf{C}} M, \xi_{\mathbf{C}}\right)$. By (2.5) and (2.8), we have

$$
\begin{aligned}
\Theta_{1}\left(T_{\mathbf{C}} M, \xi_{\mathbf{C}}\right)= & \bigotimes_{n=1}^{\infty} S_{q^{n}}\left(\widetilde{T_{\mathbf{C}} M}\right) \otimes \bigotimes_{m=1}^{\infty} \Lambda_{q^{m}}\left(\widetilde{T_{\mathbf{C}} M}-2 \widetilde{\xi_{\mathbf{C}}}\right) \\
& \otimes \bigotimes_{r=1}^{\infty}\left(\Lambda_{q^{r-\frac{1}{2}}}\left(\xi_{\mathbf{C}}\right) S_{-q^{r-\frac{1}{2}}}\left(\mathbf{C}^{2}\right)\right) \otimes\left(\bigotimes_{s=1}^{\infty} \Lambda_{-q^{s-\frac{1}{2}}}\left(\xi_{\mathbf{C}}\right) S_{q^{s-\frac{1}{2}}}\left(\mathbf{C}^{2}\right)\right) \\
= & \left(1+\left(T_{\mathbf{C}} M-(8 k+4)\right) q\right) \otimes\left(1+\left(T_{\mathbf{C}} M-(8 k+4)-2 \xi_{\mathbf{C}}+4\right) q\right) \\
& \otimes\left(1+\left(\xi_{\mathbf{C}}\right) q^{\frac{1}{2}}+\left(\xi_{\mathbf{C}} \wedge \xi_{\mathbf{C}}\right) q\right) \otimes\left(1-\mathbf{C}^{2} q^{\frac{1}{2}}+\mathbf{C}^{3} q\right) \\
& \otimes\left(1-\left(\xi_{\mathbf{C}}\right) q^{\frac{1}{2}}+\left(\xi_{\mathbf{C}} \wedge \xi_{\mathbf{C}}\right) q\right) \otimes\left(1+\mathbf{C}^{2} q^{\frac{1}{2}}+\mathbf{C}^{3} q\right)+\cdots \\
= & 1+\left[2\left(T_{\mathbf{C}} M-(8 k+4)-\xi_{\mathbf{C}}+2\right)\right. \\
& \left.\quad-\left(\xi_{\mathbf{C}} \otimes \xi_{\mathbf{C}}-2 \xi_{\mathbf{C}} \wedge \xi_{\mathbf{C}}-\mathbf{C}^{2}\right)\right] q+\cdots,
\end{aligned}
$$

where the "..." terms are the terms involving $q^{\frac{j}{2}}$ 's with $j \geq 3$.

By (5.22), (5.40) and (5.49), setting $q=0$ in (5.48), we have (9])

$$
\left\{\frac{\widehat{L}\left(T M, \nabla^{T M}\right)}{\cosh ^{2}\left(\frac{c}{2}\right)}\right\}^{(8 k+4)}=8 \sum_{r=0}^{k} 2^{6 k-6 r} h_{r}\left(T_{\mathbf{C}} M, \xi_{\mathbf{C}}\right) .
$$

On the other hand, by (5.22), (5.40) and (5.49), comparing the coefficients of $q$ in (5.48), we have

$$
\begin{aligned}
& \left\{\frac { \widehat { L } ( T M , \nabla ^ { T M } ) } { \operatorname { c o s h } ^ { 2 } ( \frac { c } { 2 } ) } \operatorname { c h } \left(2\left(T_{\mathbf{C}} M\right)-2(8 k+4)-2\left(\xi_{\mathbf{C}}-2\right)\right.\right. \\
& \left.\left.-\left(\xi_{\mathbf{C}} \otimes \xi_{\mathbf{C}}-2 \xi_{\mathbf{C}} \wedge \xi_{\mathbf{C}}-\mathbf{C}^{2}\right)\right)\right\}^{(8 k+4)} \\
= & 8 \sum_{r=0}^{k} 2^{6 k-6 r}(48 k+24-64 r) h_{r}\left(T_{\mathbf{C}} M, \xi_{\mathbf{C}}\right) .
\end{aligned}
$$

Thus similar to (5.25), we have

$$
\begin{aligned}
& \left\{\frac { \widehat { L } ( T M , \nabla ^ { T M } ) } { \operatorname { c o s h } ^ { 2 } ( \frac { c } { 2 } ) } \operatorname { c h } \left(T_{\mathbf{C}} M-(8 k+4)-\left(\xi_{\mathbf{C}}-2\right)\right.\right. \\
& \left.\left.-\frac{1}{2}\left(\xi_{\mathbf{C}} \otimes \xi_{\mathbf{C}}-2 \xi_{\mathbf{C}} \wedge \xi_{\mathbf{C}}-\mathbf{C}^{2}\right)\right)\right\}^{(8 k+4)} \\
= & 12\left(8 \sum_{r=0}^{k} 2^{6 k-6 r} h_{r}\left(T_{\mathbf{C}} M, \xi_{\mathbf{C}}\right)\right)-8 k\left(8 \sum_{r=0}^{k} 2^{6 k-6 r} h_{r}\left(T_{\mathbf{C}} M, \xi_{\mathbf{C}}\right)\right) \\
& +256 \cdot 2^{6} \sum_{r=0}^{k-1}(k-r) h_{r}\left(T_{\mathbf{C}} M, \xi_{\mathbf{C}}\right) 2^{6 k-6 r-6} .
\end{aligned}
$$


Note that

$$
\begin{aligned}
& \frac{\widehat{L}\left(T M, \nabla^{T M}\right)}{\cosh ^{2}\left(\frac{c}{2}\right)} \operatorname{ch}\left(\left(\xi_{\mathbf{C}}-2\right)+\frac{1}{2}\left(\xi_{\mathbf{C}} \otimes \xi_{\mathbf{C}}-2 \xi_{\mathbf{C}} \wedge \xi_{\mathbf{C}}-\mathbf{C}^{2}\right)\right) \\
= & \frac{\widehat{L}\left(T M, \nabla^{T M}\right)}{\cosh ^{2}\left(\frac{c}{2}\right)}\left(\left(e^{c}+e^{-c}-2\right)+\frac{1}{2}\left(\left(e^{c}+e^{-c}\right)^{2}-4\right)\right) \\
= & \frac{\widehat{L}\left(T M, \nabla^{T M}\right)}{\cosh ^{2}\left(\frac{c}{2}\right)} \sinh ^{2}\left(\frac{c}{2}\right)\left(2\left(e^{c}+e^{-c}\right)+8\right) \\
= & \frac{\widehat{L}\left(T M, \nabla^{T M}\right)}{\cosh ^{2}\left(\frac{c}{2}\right)} \sinh ^{2}\left(\frac{c}{2}\right) \operatorname{ch}\left(2 \xi_{\mathbf{C}} \oplus \mathbf{C}^{8}\right) .
\end{aligned}
$$

Therefore combining (5.50), (5.52) and (5.53), one has

$$
\begin{gathered}
\left\{\frac{\widehat{L}\left(T M, \nabla^{T M}\right)\left[\operatorname{ch}\left(T_{\mathbf{C}} M\right)-\sinh ^{2}\left(\frac{c}{2}\right) \operatorname{ch}\left(2 \xi_{\mathbf{C}} \oplus \mathbf{C}^{8}\right)-16\right]}{\cosh ^{2}\left(\frac{c}{2}\right)}\right\}^{(8 k+4)} \\
=2^{14}\left[\sum_{r=0}^{k-1}(k-r) 2^{6(k-r-1)} h_{r}\left(T_{\mathbf{C}} M, \xi_{\mathbf{C}}\right)\right],
\end{gathered}
$$

which is just (2.31).

To prove Theorem 2.6 for the $8 k$ dimensional case, similarly we set (9])

$$
P_{1}\left(\xi_{\mathbf{C}}, \tau\right)=\left\{\frac{\widehat{L}\left(T M, \nabla^{T M}\right)}{\cosh ^{2}\left(\frac{c}{2}\right)} \operatorname{ch}\left(\Theta_{1}\left(T_{\mathbf{C}} M, \xi_{\mathbf{C}}\right), \nabla^{\Theta_{1}\left(T_{\mathbf{C}} M, \xi_{\mathbf{C}}\right)}\right)\right\}^{(8 k)}
$$

$$
P_{2}\left(\xi_{\mathbf{C}}, \tau\right)=\left\{\widehat{A}\left(T M, \nabla^{T M}\right) \operatorname{ch}\left(\Theta_{2}\left(T_{\mathbf{C}} M, \xi_{\mathbf{C}}\right), \nabla^{\Theta_{2}\left(T_{\mathbf{C}} M, \xi_{\mathbf{C}}\right)}\right) \cosh \left(\frac{c}{2}\right)\right\}^{(8 k)} .
$$

Still playing the same game, we see that $P_{1}\left(\xi_{\mathbf{C}}, \tau\right)$ is a modular form of weight $4 k$ over $\Gamma_{0}(2)$, while $P_{2}\left(\xi_{\mathbf{C}}, \tau\right)$ is a modular form of weight $4 k$ over $\Gamma^{0}(2)$ and one has the following identities:

$$
P_{1}(-1 / \tau)=(2 \tau)^{4 k} P_{2}(\tau)
$$

$$
P_{2}(\tau)=z_{0}\left(T_{\mathbf{C}} M, \xi_{\mathbf{C}}\right)\left(8 \delta_{2}\right)^{2 k}+z_{1}\left(T_{\mathbf{C}} M, \xi_{\mathbf{C}}\right)\left(8 \delta_{2}\right)^{2 k-2} \varepsilon_{2}+\cdots+z_{k}\left(T_{\mathbf{C}} M, \xi_{\mathbf{C}}\right) \varepsilon_{2}^{k} .
$$

Thus

(5.59)

$P_{1}(\tau)=2^{4 k}\left[z_{0}\left(T_{\mathbf{C}} M, \xi_{\mathbf{C}}\right)\left(8 \delta_{1}\right)^{2 k}+z_{1}\left(T_{\mathbf{C}} M, \xi_{\mathbf{C}}\right)\left(8 \delta_{1}\right)^{2 k-2} \varepsilon_{1}+\cdots+z_{k}\left(T_{\mathbf{C}} M, \xi_{\mathbf{C}}\right) \varepsilon_{1}^{k}\right]$.

By direct computations, we have

$$
\begin{gathered}
z_{0}\left(T_{\mathbf{C}} M, \xi_{\mathbf{C}}\right)=\left\{\widehat{A}\left(T M, \nabla^{T M}\right)\right\}^{(8 k)} \\
z_{1}\left(T_{\mathbf{C}} M, \xi_{\mathbf{C}}\right)=-\left\{\widehat{A}\left(T M, \nabla^{T M}\right)\left[48 k-\operatorname{ch}\left(B_{1}\left(T_{\mathbf{C}} M, \xi_{\mathbf{C}}\right)\right)\right]\right\}^{(8 k)} .
\end{gathered}
$$

As we did in (5.49), explicitly expanding $\Theta_{1}\left(T_{\mathbf{C}} M, \xi_{\mathbf{C}}\right)$, we get $(5.62) \Theta_{1}\left(T_{\mathbf{C}} M, \xi_{\mathbf{C}}\right)=1+\left[2\left(T_{\mathbf{C}} M-(8 k)-\xi_{\mathbf{C}}+2\right)-\left(\xi_{\mathbf{C}} \otimes \xi_{\mathbf{C}}-2 \xi_{\mathbf{C}} \wedge \xi_{\mathbf{C}}-\mathbf{C}^{2}\right)\right] q+\cdots$, where the "..." terms are the terms involving $q^{\frac{j}{2}}$ 's with $j \geq 3$. 
By (5.35), (5.55) and (5.62), setting $q=0$ in (5.59), we have ( 8 , 9$]$ )

$$
\left\{\frac{\widehat{L}\left(T M, \nabla^{T M}\right)}{\cosh ^{2}\left(\frac{c}{2}\right)}\right\}^{(8 k)}=\sum_{r=0}^{k} 2^{6 k-6 r} z_{r}\left(T_{\mathbf{C}} M, \xi_{\mathbf{C}}\right) .
$$

By (5.35), (5.55) and (5.62), comparing the coefficients of $q$ in (5.59), we have

$$
\begin{aligned}
& \begin{aligned}
\left\{\frac { \widehat { L } ( T M , \nabla ^ { T M } ) } { \operatorname { c o s h } ^ { 2 } ( \frac { c } { 2 } ) } \operatorname { c h } \left(2\left(T_{\mathbf{C}} M, \nabla^{T_{\mathbf{C}} M}\right)\right.\right. & -2 \cdot 8 k-2\left(\xi_{\mathbf{C}}-2\right) \\
& \left.\left.-\left(\xi_{\mathbf{C}} \otimes \xi_{\mathbf{C}}-2 \xi_{\mathbf{C}} \wedge \xi_{\mathbf{C}}-\mathbf{C}^{2}\right)\right)\right\}^{(8 k)} \\
= & \sum_{r=0}^{k} 2^{6 k-6 r}(48 k-64 r) z_{r}\left(T_{\mathbf{C}} M, \xi_{\mathbf{C}}\right) .
\end{aligned}
\end{aligned}
$$

Thus similar to (5.38), we have

$$
\begin{aligned}
\left\{\frac { \widehat { L } ( T M , \nabla ^ { T M } ) } { \operatorname { c o s h } ^ { 2 } ( \frac { c } { 2 } ) } \operatorname { c h } \left(\left(T_{\mathbf{C}} M, \nabla^{T_{\mathbf{C}} M}\right)\right.\right. & -8 k-\left(\xi_{\mathbf{C}}-2\right) \\
& \left.\left.-\frac{1}{2}\left(\xi_{\mathbf{C}} \otimes \xi_{\mathbf{C}}-2 \xi_{\mathbf{C}} \wedge \xi_{\mathbf{C}}-\mathbf{C}^{2}\right)\right)\right\}^{(8 k)} \\
=-8 k\left(\sum_{r=0}^{k} 2^{6 k-6 r} z_{r}\left(T_{\mathbf{C}} M, \xi_{\mathbf{C}}\right)\right) & +32 \cdot 2^{6} \sum_{r=0}^{k-1}(k-r) 2^{6 k-6 r-6} z_{r}\left(T_{\mathbf{C}} M, \xi_{\mathbf{C}}\right) .
\end{aligned}
$$

Combining (5.63), (5.65) and (5.53), one has

$$
\begin{gathered}
\left\{\frac{\widehat{L}\left(T M, \nabla^{T M}\right)\left[\operatorname{ch}\left(T_{\mathbf{C}} M, \nabla^{T_{\mathbf{C}} M}\right)-\sinh ^{2}\left(\frac{c}{2}\right) \operatorname{ch}\left(2 \xi_{\mathbf{C}} \oplus \mathbf{C}^{8}\right)\right]}{\cosh ^{2}\left(\frac{c}{2}\right)}\right\}^{(8 k)} \\
=2^{11}\left[\sum_{r=0}^{k-1}(k-r) 2^{6(k-r-1)} z_{r}\left(T_{\mathbf{C}} M, \xi_{\mathbf{C}}\right)\right],
\end{gathered}
$$

which is just (2.32).

Remark 5.1. Our main results are obtained by comparing the coefficients of $q$ and $q^{2}$ in (5.20), (5.31) and the coefficients of $q$ in (5.48), (5.59). It is interesting to examine other coefficients of higher powers of $q$ to get further divisibility and congruence results. These will be developed elsewhere.

\section{ACKNOWLEDGMENTS}

We are grateful to Professor Weiping Zhang for helpful suggestions and inspiring discussions with us. We also thank Professor Peter Teichner for giving us many suggestions and offering us a lot of help. Professor M. Atiyah and Professor F. Hirzebruch are appreciated for communications with us. The first author thanks Professor Nicolai Reshetikhin for his interest, encouragement and support. 


\section{REFERENCES}

1. L. Alvarez-Gaumé and E. Witten, Gravitational anomalies. Nucl. Phys. B234 (1983), 269-330. MR.736803 (85j:81062)

2. M. F. Atiyah, K-theory. Benjamin, New York, 1967. MR0224083 (36:7130)

3. M. F. Atiyah and F. Hirzebruch, Riemann-Roch theorems for differentiable manifolds. Bull. Amer. Math. Soc. 65 (1959), 276-281. MR0110106 (22:989)

4. M. F. Atiyah and I. M. Singer, The index of elliptic operators, III, Ann. Math. 87 (1968), 546-604. MR0236952 (38:5245)

5. K. Chandrasekharan, Elliptic Functions. Springer-Verlag, 1985. MR808396 (87e:11058)

6. S. M. Finashin, A Pin ${ }^{-}$-cobordism invariant and a generalization of Rokhlin signature congruence. Leningrad Math. J. 2 (1991), 917-924. MR1080207 (91i:57016)

7. Peter B. Gilkey, Invariance Theory, the Heat Equation and the Atiyah-Singer Index Theorem, Second Edition. CRC Press, Inc., 1995. MR1396308 (98b:58156)

8. F. Han and W. Zhang, Spin ${ }^{c}$-manifold and elliptic genera. C. R. Acad. Sci. Paris, Série I. 336 (2003), 1011-1014. MR.1993972 (2004j:58024)

9. F. Han and W. Zhang, Modular invariance, characteristic numbers and $\eta$ invariants. Journal of Differential Geometry. 67 (2004), 257-288. MR2153079 (2006k:58038)

10. F. Hirzebruch, T. Berger and R. Jung, Manifolds and Modular Forms. Aspects of Mathematics, vol. E20, Viehweg, Braunschweig, 1992. MR1189136 (94d:57001)

11. F. Hirzebruch, Topological Methods in Algebraic Geometry. Springer-Verlag, 1966. MR0202713 (34:2573)

12. F. Hirzebruch, Mannigfaltigkeiten und Modulformen. Jahresberichte der Deutschen Mathematiker Vereinigung, Jber. d. Dt. Math.-Verein, 1992, pp. 20-38.

13. Boyuan Hou and Boyu Hou, Differential Geomerty for Physicists, Second Edition (in Chinese). Science Press, China, 2004.

14. P. S. Landweber, Elliptic cohomology and modular forms. in Elliptic Curves and Modular Forms in Algebraic Topology, pp. 55-68. Ed. P. S. Landweber. Lecture Notes in Mathematics Vol. 1326, Springer-Verlag (1988). MR.970281

15. G. Laures, K(1)-local topological modular forms. Invent. Math. (2004), 371-403. MR 2076927 (2005h:55003)

16. K. Liu, Modular invariance and characteristic numbers. Commun. Math. Phys. 174 (1995), 29-42. MR:1372798 (96m:57034)

17. K. Liu, On Modular Invariance and Rigidity Theorems, Ph.D. Dissertation at Harvard University. 1993.

18. K. Liu and W. Zhang, Elliptic genus and $\eta$-invariants. Inter. Math. Res. Notices No. 8 (1994), 319-328. MR1289577 (96b:57030)

19. S. Ochanine, Signature modulo 16, invariants de Kervaire généralisés et nombres caractéristiques dans la K-théorie réelle. Mémoire Soc. Math. France, Tom. 109 (1980/81), 1-141. MR:0615511 (83j:57014)

20. W. Zhang, Spin ${ }^{c}$-manifolds and Rokhlin congruences. C. R. Acad. Sci. Paris, Série I, 317 (1993), 689-692. MR.1245100 (94i:57042)

21. W. Zhang, Circle bundles, adiabatic limits of $\eta$-invariants and Rokhlin congruences. Ann. Inst. Fourier 44 (1994), 249-270. MR1262887 (95h:58127)

22. W. Zhang, Lectures on Chern-Weil Theory and Witten Deformations. Nankai Tracts in Mathematics Vol. 4, World Scientific, River Edge, NJ, 2001. MR.1864735 (2002m:58032)

Department of Mathematics, University of California, Berkeley, California 947203840

E-mail address: chenqtao@math.berkeley.edu

Department of Mathematics, University of California, Berkeley, California 947203840

E-mail address: feihan@math.berkeley.edu

Current address: Department of Mathematics, Stanford University, Stanford, California 943052125 